\title{
Global Classical Solutions to the Mixed Initial-boundary Value Problem for a Class of Quasilinear Hyperbolic Systems of Balance Laws
}

\section{Zhi-Qiang Shao*}

Department of Mathematics, Fuzhou University, Fuzhou 350002, China

\begin{abstract}
It is proven that the mixed initial-boundary value problem for a class of quasilinear hyperbolic systems of balance laws with general nonlinear boundary conditions in the half space $\mid\{(t, x) \mid t \geq 0, x \geq 0\}$ admits a unique global C1 solution $\mathrm{u}=\mathrm{u}(\mathrm{t}, \mathrm{x})$ with small $\mathrm{C} 1$ norm, provided that each characteristic with positive velocity is weakly linearly degenerate. This result is also applied to the flow equations of a model class of fluids with viscosity induced by fading memory.
\end{abstract}

MSC: 35L45; 35L50; 35Q72.

Keywords: Mixed initial-boundary value problem; Global classical solution; Quasilinear hyperbolic; systems of balance laws; Weakly linearly degenerate characteristics

\section{Introduction and Main Result}

Consider the following quasilinear hyperbolic system of balance laws in one space dimension:

$$
\frac{\partial \mathrm{u}}{\partial \mathrm{t}}+\frac{\partial \mathrm{f}(\mathrm{u})}{\partial \mathrm{x}}+\mathrm{Lu}=0
$$

where $\mathrm{L}>0$ is a constant; $\mathrm{u}=\left(\mathrm{u}^{1}, \ldots, \mathrm{u}^{\mathrm{n}}\right)^{\mathrm{T}}$ is the unknown vector function of $(t, x), f(u)$ is a given $C^{3}$ vector function of $u$.

It is assumed that system (1.1) is strictly hyperbolic, i.e., for any given $\mathrm{u}$ on the domain under consideration, the Jacobian $\mathrm{A}(\mathrm{u})=\nabla \mathrm{f}(\mathrm{u})$ has $\mathrm{n}$ real distinct eigenvalues.

$$
\lambda_{1}(\mathrm{u})<\lambda_{2}(\mathrm{u})<\ldots<\lambda_{\mathrm{n}}(\mathrm{u})
$$

Let $1_{\mathrm{i}}(\mathrm{u})=\left(\mathrm{l}_{\mathrm{i} 1}(\mathrm{u}), \ldots \mathrm{l}_{\text {in }}(\mathrm{u})\right)\left(\operatorname{resp} . \mathrm{r}_{\mathrm{i}}(\mathrm{u})=\left(\mathrm{r}_{\mathrm{i}}(\mathrm{u}), \ldots, \mathrm{r}_{\text {in }}(\mathrm{u})\right)^{\mathrm{T}}\right)$ be a left (resp. right) eigenvector corresponding to $\lambda \mathrm{i}(\mathrm{u})(\mathrm{i}=1, \ldots, \mathrm{n})$

$1_{\mathrm{i}}(\mathrm{u}) \mathrm{A}(\mathrm{u})=\lambda_{\mathrm{i}}(\mathrm{u}) \mathrm{l}_{\mathrm{i}}(\mathrm{u})\left(\operatorname{resp} \cdot \mathrm{A}(\mathrm{u}) \mathrm{r}_{\mathrm{i}}(\mathrm{u})=\lambda_{\mathrm{i}}(\mathrm{u}) \mathrm{r}_{\mathrm{i}}(\mathrm{u})\right)$

then we have

$\operatorname{det}\left|1_{\mathrm{ij}}(\mathrm{u})\right| \neq 0$ (Equivalently, $\operatorname{det}\left|1_{\mathrm{ij}}(\mathrm{u})\right| \neq 0$ ).

Without loss of generality, we may assume that on the domain under consideration

$$
1_{i}(u) r_{j}(u) \equiv \delta_{i j}(i, j=1, \ldots, n)
$$

And

$$
r_{i}^{\mathrm{T}}(\mathrm{u}) \mathrm{r}_{\mathrm{i}}(\mathrm{u}) \equiv 1 \quad(\mathrm{i}=1, \ldots ., \mathrm{n})
$$

Where $\delta_{\mathrm{ij}}$ stands for the Kronecker's symbol.

Clearly, all $\lambda_{\mathrm{i}}(\mathrm{u}), 1_{\mathrm{ij}}(\mathrm{u})$ and $\mathrm{r}_{\mathrm{ij}}(\mathrm{u})(\mathrm{i}, \mathrm{j}=1, \ldots, \mathrm{n})$

have the same regularity as $\mathrm{A}(\mathrm{u})$, i.e., $\mathrm{C}^{2}$ regularity.

We assume that on the domain under consideration, each characteristic with positive velocity is weakly linearly degenerate and the eigenvalues of $\mathrm{A}(\mathrm{u})=\nabla \mathrm{f}(\mathrm{u})$

satisfy the non-characteristic condition.

$$
\lambda \mathrm{r}(\mathrm{u})<0<\lambda \mathrm{s}(\mathrm{u})
$$

$$
(\mathrm{r}=1, \ldots ., \mathrm{m} ; \mathrm{s}=\mathrm{m}+1, \ldots, \mathrm{n})
$$

We are concerned with the existence and uniqueness of global $\mathrm{C}^{1}$ solutions to the mixed initial-boundary value problem for system (1.1) in the half space

$$
\mathrm{D}=\{(\mathrm{t}, \mathrm{x}) \mid \mathrm{t} \geq 0, \mathrm{x} \geq 0\}
$$

with the initial condition:

$\mathrm{t}=0: \mathrm{u}=\varphi(\mathrm{x})(\mathrm{x} \geq 0)$

and the nonlinear boundary condition:

$\mathrm{x}=0: \mathrm{v}_{\mathrm{s}}=\mathrm{G}_{\mathrm{s}}(\alpha(\mathrm{t}), \mathrm{v} 1 \ldots \mathrm{vm})+\mathrm{h}_{\mathrm{s}}(\mathrm{t}), \mathrm{s}=\mathrm{m}+1, \ldots, \mathrm{n}(\mathrm{t} \geq 0)$

Where

$\mathrm{v}_{\mathrm{i}}=1_{\mathrm{i}}(\mathrm{u}) \mathrm{u}(\mathrm{i}=1, \ldots, \mathrm{n})$

And

$\alpha(\mathrm{t})=\left(\alpha_{1}(\mathrm{t}), \ldots, \alpha_{\mathrm{k}}(\mathrm{t})\right)$

Here, $\varphi=\left(\varphi_{1}, \ldots, \varphi_{\mathrm{n}}\right)^{\mathrm{T}}, \alpha, \mathrm{G}_{\mathrm{s}}$ and $\mathrm{h}_{\mathrm{s}}(\mathrm{s}=\mathrm{m}+1, \ldots ., \mathrm{n})$ are all $\mathrm{C}^{1}$ functions with respect to their arguments, which satisfy the conditions of $\mathrm{C}^{1}$ compatibility at the point $(0 ; 0)$. Also, we assume that there exists a constant $\mu>0$ such that

$$
\begin{aligned}
& \theta \triangleq \max \left\{\sup _{\mathrm{x} \geq 0}(1+\mathrm{x}) 1+\mu\left(|\varphi(\mathrm{x})|+\left|\varphi^{\prime}(\mathrm{x})\right| \sup _{\mathrm{t} \geq 0}\right.\right. \\
& \left.(1+\mathrm{t}) 1+\mu\left(|\varphi(\mathrm{t})|+|\mathrm{h}(\mathrm{t})|+|\alpha(\mathrm{t})|+\left|\alpha^{\prime}(\mathrm{t})\right|+\left|\mathrm{h}^{\prime}(\mathrm{t})\right|\right)\right\}<+\infty
\end{aligned}
$$

in which

$h(t)=\left(h_{m+1}(t), \ldots ., h_{n}(t)\right)$

*Corresponding author: Zhi-Qiang Shao, Department of Mathematics Fuzhou University, Fuzhou 350002, China, Tel: 86-0591-83852790; E-mail: zqshaojfu@yahoo.com.cn

Received November 24, 2014; Accepted November 25, 2014; Published December 10, 2014

Citation: Shao ZQ (2014) Global Classical Solutions to the Mixed Initial-boundary Value Problem for a Class of Quasilinear Hyperbolic Systems of Balance Laws. J Appl Computat Math 3: 197. doi:10.4172/2168-9679.1000197

Copyright: (c) 2014 Shao ZQ. This is an open-access article distributed under the terms of the Creative Commons Attribution License, which permits unrestricted use, distribution, and reproduction in any medium, provided the original author and source are credited. 
Citation: Shao ZQ (2014) Global Classical Solutions to the Mixed Initial-boundary Value Problem for a Class of Quasilinear Hyperbolic Systems of Balance Laws. J Appl Computat Math 4: 197. doi:10.4172/2168-9679.1000197

Without loss of generality, we assume that

$$
\mathrm{G}_{\mathrm{s}}(\alpha(\mathrm{t}), \ldots, 0) \equiv 0(\mathrm{~s}=\mathrm{m}+1, \ldots, \mathrm{n})
$$

For the special case where (1.1) is a quasilinear hyperbolic system of conservation laws, i.e., $\mathrm{L}=0$, such kinds of problems have been extensively studied (for instance, [1-8] and the references therein). In particular, Li and Wang proved the existence and uniqueness of global $\mathrm{C}^{1}$ solutions to the mixed initial boundary value problem for first order quasilinear hyperbolic systems with general nonlinear boundary conditions in the half space $\{(\mathrm{t}, \mathrm{x}|\mathrm{t} \geq 0, \mathrm{x} \geq 0|)\}$. On the other hand, for quasilinear hyperbolic systems of balance laws, many results on the existence of global solutions have also been obtained by Liu, et al., (for instance, see [8-14] and the references therein), and some methods have been established. So the following question arises naturally: when can we obtain the existence and uniqueness of semiglobal C1 solutions for quasilinear hyperbolic systems of balance laws? It is well known that for first-order quasilinear hyperbolic systems of balance laws, generically speaking, the classical solution exists only locally in time and the singularity will appear in a finite time even if the data are sufficiently smooth and small [15-20]. However, in some cases global existence in time of classical solutions can be obtained. In this paper, we will generalize the results in [21] to a nonhomogeneous quasilinear hyperbolic system, the analysis relies on a careful study of the interaction of the nonhomogeneous term. Our main results can be stated as follows:

Theorem 1.1. Suppose that the non-characteristic condition (1.10) holds and system (1.1) is strictly hyperbolic. Suppose furthermore that for $\mathrm{j}=\mathrm{m}+1, \ldots ., \mathrm{n}$; each $\mathrm{j}$-characteristic field with positive velocity is weakly linearly degenerate. Suppose finally that $\varphi, \alpha, \mathrm{G}_{\mathrm{s}}, \mathrm{h}_{\mathrm{s}}(\mathrm{s}=\mathrm{m}+1, \ldots, \mathrm{n})$ are all $\mathrm{C}^{1}$ functions with respect to their arguments, satisfying (1.15)-(1.16) and the conditions of $\mathrm{C}^{1}$ compatibility at the point $(0 ; 0)$. Then there exists a sufficiently small $\theta_{0}>0$ such that for any given $\theta \in\left[0, \theta_{0}\right]$ the mixed initial-boundary value problem (1.1) and (1.12)-(1.13) admits a unique global $\mathrm{C} 1$ solution $\mathrm{u}=\mathrm{u}(\mathrm{t} ; \mathrm{x})$ in the half space $\{(\mathrm{t}, \mathrm{x}) \mid \mathrm{t} \geq 0, \mathrm{x} \geq 0\}$.

The rest of this paper is organized as follows. In Section 2, we give the main tools of the proof that is several formulas on the decomposition of waves for system (1.1). Then, the main result will be proved in Section 3. Finally, an application is given in Section 4 [22].

\section{Decomposition of Waves}

Suppose that on the domain under consideration, system (1.1) is strictly hyperbolic and (1.2)-(1.6) hold.

Suppose that $\mathrm{A}(\mathrm{u}) \in \mathrm{C}^{\mathrm{k}}$ where $\mathrm{k}$ is an integer , 1. By Lemma 2.5 in [23], there exists an invertible $C^{\mathrm{k}+1}$ transformation $\mathrm{u}=\mathrm{u}(\tilde{\mathrm{u}})(\mathrm{u}(0)=0)$ such that in $\tilde{u}$-space for each $i=1, \ldots, n$, the ith characteristic trajectory passing through $\tilde{\mathrm{u}}=0$ coincides with the $\tilde{\mathrm{u}}_{\mathrm{i}}$-axis at least for $\left|\tilde{\mathrm{u}}_{\mathrm{i}}\right|$ small, namely,

$$
\tilde{\mathrm{r}}_{\mathrm{i}}\left(\tilde{\mathrm{u}}_{\mathrm{i}} \mathrm{e}_{\mathrm{i}}\right) \equiv \mathrm{ei}, \forall|\tilde{\mathrm{u}} \mathrm{i}| \operatorname{small}(\mathrm{i}=1, \ldots ., \mathrm{n}) \text {; }
$$

Where

$$
\mathrm{e}_{\mathrm{i}}=(0, \ldots, 0, \stackrel{(\mathrm{i})}{1}, 0, \ldots, 0)^{\mathrm{T}}
$$

This transformation is called the normalized transformation, and the corresponding unknown variables $\tilde{\mathrm{u}}=(\tilde{\mathrm{u}} 1, \ldots, \tilde{u} n)^{\mathrm{T}}$ are called the normalized variables or normalized coordinates [24-28].

\section{Let}

$w i=l i(u) u x(i=1, \ldots, n)$; where

$l i(u)=(l i 1(u) ;:: ; \operatorname{lin}(u))$

denotes the ith left eigenvector.

By (1.5), it follows from (1.14) and (2.2) that

$\mathrm{u}=\sum_{\mathrm{k}=1}^{\mathrm{n}} \mathrm{v}_{\mathrm{k}} \mathrm{r}_{\mathrm{k}}(\mathrm{u})$

And

$\mathrm{u}_{\mathrm{x}}=\sum_{\mathrm{k}=1}^{\mathrm{n}} \mathrm{w}_{\mathrm{k}} \mathrm{r}_{\mathrm{k}}(\mathrm{u})$

Let

$\frac{\mathrm{d}}{\mathrm{d}_{\mathrm{i}} \mathrm{t}}=\frac{\partial}{\partial \mathrm{t}}+\lambda_{\mathrm{i}}(\mathrm{u}) \frac{\partial}{\partial \mathrm{x}}$

be the directional derivative along the ith characteristic. Our aim in this section is to prove several formulas on the decomposition of waves for system (1.1), which will play an important role in our discussion.

\section{Lemma 2.1.}

$\frac{\mathrm{d}\left(\mathrm{e}^{\mathrm{Lt}} \mathrm{w}_{\mathrm{i}}\right)}{\mathrm{d}_{\mathrm{i}} \mathrm{t}}=\sum_{\mathrm{j}, \mathrm{k}=1}^{\mathrm{n}} \mathrm{e}^{\mathrm{Lt}} \gamma_{\mathrm{ijk}}(\mathrm{u}) \mathrm{w}_{\mathrm{j}} \mathrm{w}_{\mathrm{k}}+\sum_{\mathrm{j}, \mathrm{k}=1}^{\mathrm{n}} \mathrm{e}^{\mathrm{Lt} \tau} \gamma_{\mathrm{ijk}}(\mathrm{u}) \mathrm{v}_{\mathrm{j}} \mathrm{w}_{\mathrm{k}}(\mathrm{i}=1, \ldots, \mathrm{n})$

Where

$$
\begin{aligned}
& \gamma_{\mathrm{ijk}}(\mathrm{u})=\left(\lambda_{\mathrm{k}}(\mathrm{u})-\lambda_{\mathrm{j}}(\mathrm{u}) \mathrm{r}_{\mathrm{j}}^{\mathrm{T}}(\mathrm{u}) \nabla \mathrm{l}_{\mathrm{i}}(\mathrm{u}) \mathrm{r}_{\mathrm{k}}(\mathrm{u})-\nabla \lambda_{\mathrm{i}}(\mathrm{u}) \mathrm{r}_{\mathrm{j}}(\mathrm{u}) \delta_{\mathrm{ik}}\right) \\
& \tilde{\gamma}_{\mathrm{ijk}}(\mathrm{u})=-\mathrm{Lr}_{\mathrm{j}}^{\mathrm{T}}(\mathrm{u}) \nabla 1_{\mathrm{i}}(\mathrm{u}) \mathrm{r}_{\mathrm{k}}(\mathrm{u})
\end{aligned}
$$

Hence, we have

$$
\begin{aligned}
& \tilde{\gamma}_{\mathrm{ijk}}(\mathrm{u}) \equiv 0, \forall \mathrm{j} \neq \mathrm{i}(\mathrm{i}, \mathrm{j}=1, \ldots ., \mathrm{n}) \\
& \gamma_{\mathrm{iii}}(\mathrm{u})=-\nabla \lambda_{\mathrm{i}}(\mathrm{u}) \mathrm{r}_{\mathrm{i}}(\mathrm{u})(\mathrm{i}=1, \ldots ., \mathrm{n})
\end{aligned}
$$

Moreover, in the normalized coordinates,

$$
\tilde{\gamma}_{\mathrm{ijj}}\left(\mathrm{u}_{\mathrm{j}} \mathrm{e}_{\mathrm{j}}\right) \equiv 0, \forall\left|\mathrm{u}_{\mathrm{j}}\right| \text { small, } \forall \mathrm{i}, \mathrm{j} \text {; }
$$

while, when the ith characteristic $\lambda_{\mathrm{i}}(\mathrm{u})$ is weakly linearly degenerate, in the normalized coordinates,

$\gamma_{\text {iii }}\left(\mathrm{u}_{\mathrm{i}} \mathrm{e}_{\mathrm{i}}\right) \equiv 0, \forall\left|\mathrm{u}_{\mathrm{i}}\right|$ small, $\forall \mathrm{i}$.

Lemma 2.2 .

$$
\frac{d\left(e^{\mathrm{Lt}} \mathrm{v}_{\mathrm{i}}\right)}{\mathrm{d}_{\mathrm{i}} \mathrm{t}}=\sum_{\mathrm{j}, \mathrm{k}=1}^{\mathrm{n}} \mathrm{e}^{\mathrm{Lt}} \beta_{\mathrm{ijk}}(\mathrm{u}) \mathrm{v}_{\mathrm{j}} \mathrm{w}_{\mathrm{k}}+\sum_{\mathrm{j}, \mathrm{k}=1}^{\mathrm{n}} \mathrm{e}^{\mathrm{Lt}} \tilde{\beta}_{\mathrm{ijk}}(\mathrm{u}) \mathrm{v}_{\mathrm{j}} \mathrm{v}_{\mathrm{k}}(\mathrm{i}=1, \ldots, \mathrm{n})
$$

Where

$$
\begin{aligned}
& \beta_{\mathrm{ijk}}(\mathrm{u})=\left(\lambda_{\mathrm{i}}(\mathrm{u})\right) \mathrm{r}_{\mathrm{j}}^{\mathrm{T}}(\mathrm{u}) \nabla \operatorname{li}(\mathrm{u}) \mathrm{r}_{\mathrm{k}}(\mathrm{u}) \\
& \tilde{\beta}_{\mathrm{ijk}}(\mathrm{u})=-\operatorname{Lr}_{\mathrm{j}}^{\mathrm{T}}(\mathrm{u}) \nabla \operatorname{li}(\mathrm{u}) \mathrm{r}_{\mathrm{k}}(\mathrm{u})
\end{aligned}
$$

Thus, we have

$$
\tilde{\beta}_{\mathrm{ijk}}(\mathrm{u}) \equiv 0, \quad \forall \mathrm{i}, \mathrm{j}(\mathrm{i}, \mathrm{j}=1, \ldots, \mathrm{n})
$$

Moreover, by (2.1), in the normalized coordinates we have 
$\beta_{\mathrm{ijk}}\left(\mathrm{u}_{\mathrm{j}} \mathrm{e}_{\mathrm{j}}\right) \equiv 0, \quad \forall\left|\mathrm{u}_{\mathrm{j}}\right|$ small, $\forall \mathrm{i}, \mathrm{j}$

And

$\tilde{\beta}_{\mathrm{ijk}}\left(\mathrm{u}_{\mathrm{j}} \mathrm{e}_{\mathrm{j}}\right) \equiv 0, \quad \forall\left|\mathrm{u}_{\mathrm{j}}\right|$ small, $\forall \mathrm{i}, \mathrm{j}$

The proofs of Lemmas 2.1-2.2 can be found in [29].

Similarly, we have

Lemma 2.3. In the normalized coordinates, it follows that

$$
\frac{d\left(e^{L t} u_{i}\right)}{d_{i} t}=\sum_{j, k=1}^{n} e^{L t} p_{i j k}(u) u_{j} w_{k}(i=1, \ldots ., n)
$$

Where

$$
\mathrm{p}_{\mathrm{ijj}}(\mathrm{u})=0, \forall \mathrm{i}, \mathrm{j}
$$

And

$$
\mathrm{p}_{\mathrm{ijj}}(\mathrm{u})=\left(\lambda_{\mathrm{k}}(\mathrm{u})\right) \int_{0}^{1} \frac{\partial \mathrm{r}_{\mathrm{ki}}}{\partial \mathrm{u}_{\mathrm{j}}}\left(\mathrm{Tu} 1, \ldots \ldots, \mathrm{Tu}_{\mathrm{k}-1,} \mathrm{u}_{\mathrm{k}+1}, \ldots, \mathrm{Tu}_{\mathrm{n}}\right) \mathrm{dT}
$$

Hence,

$$
\mathrm{p}_{\mathrm{ijj}}(\mathrm{u}) \equiv 0, \quad \forall \mathrm{i}, \mathrm{j}
$$

Proof. By (1.1), (2.4) and (2.5), it is easy to see that

$\frac{\mathrm{du}}{\mathrm{d}_{\mathrm{i}} \mathrm{t}}=\sum_{\mathrm{k}=1}^{\mathrm{n}}\left(\lambda_{\mathrm{i}}(\mathrm{u})-\lambda_{\mathrm{k}}(\mathrm{u})\right) \mathrm{w}_{\mathrm{k}} \mathrm{r}_{\mathrm{k}}(\mathrm{u})-\mathrm{Lu}$

On the other hand, we have

$$
\mathrm{u}_{\mathrm{i}}(\mathrm{t}, \mathrm{x})=\mathrm{u}^{\mathrm{T}}(\mathrm{t}, \mathrm{x}) \mathrm{e}_{\mathrm{i}}
$$

Thus, noting (2.1), in the normalized coordinates, it follows from (2.23)-(2.24) that

$$
\frac{d u_{i}}{d_{i} t}=\sum_{k=1}^{n}\left(\lambda_{i}(u)-\lambda_{k}(u)\right) w_{k} r_{k}^{T}(u) e_{i}-L u_{i}=\sum_{k=1}^{n}\left(\lambda_{i}(u)-\lambda_{k}(u)\right) w_{k}\left[r_{k}^{T}(u)-r_{k}^{T}\left(u_{k} e_{k}\right)\right] e_{i}-L u_{i}
$$

By Hadamard's formula, we have

$$
\mathrm{r}_{\mathrm{k}}^{\mathrm{T}}(\mathrm{u})-\mathrm{r}_{\mathrm{k}}^{\mathrm{T}}\left(\mathrm{u}_{\mathrm{k}} \mathrm{e}_{\mathrm{k}}\right)=\int_{0}^{1} \sum_{\mathrm{j} \neq \mathrm{k}} \frac{\partial \mathrm{r}_{\mathrm{k}}^{\mathrm{T}}}{\partial \mathrm{u}_{\mathrm{j}}}\left(\mathrm{Tu}_{1}, \ldots \mathrm{Tu}_{\mathrm{k}-1}, \mathrm{u}_{\mathrm{k}}, \mathrm{Tu}_{\mathrm{k}+1}, \ldots, \mathrm{Tu}_{\mathrm{n}}\right) \mathrm{u}_{\mathrm{j}} \mathrm{ds}
$$

Therefore, from (2.25)-(2.26) we immediately get (2.19)-(2.22). The proof of Lemma 2.3 is finished.

For any given $\mathrm{y} \geq 0$ on the existence domain of $C^{1}$ solution, let $\mathrm{x}=\tilde{\mathrm{x}}_{\mathrm{i}}(\mathrm{t}, \mathrm{y})$ be the ith characteristic passing through point $\left(\frac{\mathrm{y}}{\mathrm{a}} ; y\right)(a$
$>0$, constant):

$$
\left\{\begin{array}{l}
\frac{\mathrm{d} \tilde{x}_{\mathrm{i}}(\mathrm{t}, \mathrm{y})}{\mathrm{dt}}=\lambda_{\mathrm{i}}\left(\mathrm{u}\left(\mathrm{t}, \tilde{\mathrm{x}}_{\mathrm{i}}(\mathrm{t}, \mathrm{y})\right)\right) \\
\tilde{\mathrm{x} i}\left(\frac{\mathrm{y}}{\mathrm{a}}, \mathrm{y}\right)=\mathrm{y}
\end{array}\right.
$$

Lemma 2.4. Let $q i(t ; x)$ be defined by $\mathrm{q}_{\mathrm{i}}\left(\mathrm{t}, \tilde{\mathrm{x}}_{\mathrm{i}}(\mathrm{t}, \mathrm{y})\right)=\mathrm{w}_{\mathrm{i}}\left(\mathrm{t}, \tilde{\mathrm{x}}_{\mathrm{i}}(\mathrm{t}, \mathrm{y})\right) \frac{\partial \tilde{\mathrm{x}}_{\mathrm{i}}(\mathrm{t}, \mathrm{y})}{\partial \mathrm{y}}$ Then along the ith characteristic

$$
\begin{aligned}
& \mathrm{x}=\tilde{\mathrm{x}}_{\mathrm{i}}(\mathrm{t}, \mathrm{y}) \text { we have } \\
& \frac{\mathrm{d}\left(\mathrm{e}^{\mathrm{Lt}} \mathrm{q}_{\mathrm{i}}\right)}{\mathrm{d}_{\mathrm{i}} \mathrm{t}}=\sum_{\mathrm{j}, \mathrm{k}=1}^{\mathrm{n}} \mathrm{e}^{\mathrm{Lt}} \Gamma_{\mathrm{ijk}}(\mathrm{u}) \frac{\partial \mathrm{x}_{\mathrm{i}}(\mathrm{t}, \mathrm{y})}{\partial \mathrm{y}} \mathrm{w}_{\mathrm{j}} \mathrm{w}_{\mathrm{k}}+\sum_{\mathrm{j}, \mathrm{k}=1}^{\mathrm{n}} \mathrm{e}^{\mathrm{Lt}} \tilde{\gamma} \mathrm{\gamma jk}(\mathrm{u}) \frac{\partial \tilde{\mathrm{x}}_{\mathrm{i}}(\mathrm{t}, \mathrm{y})}{\partial \mathrm{y}} \mathrm{v}_{\mathrm{j}} \mathrm{w}_{\mathrm{k}}
\end{aligned}
$$

Where $\tilde{\gamma} \mathrm{ijk}(\mathrm{u})$ is given by $(2.8)$ and

$$
\Gamma_{\mathrm{ijk}}(\mathrm{u})=\left(\lambda_{\mathrm{k}}(\mathrm{u})-\lambda_{\mathrm{j}}(\mathrm{u})\right) \mathrm{r}_{\mathrm{j}}^{\mathrm{T}}(\mathrm{u}) \nabla 1_{\mathrm{i}}(\mathrm{u}) \mathrm{r}_{\mathrm{k}}(\mathrm{u})
$$

Hence,

$$
\Gamma_{\mathrm{ijk}}(\mathrm{u})=0 \quad \forall \mathrm{i}, \mathrm{j}
$$

Proof. Differentiating the first equation of (2.27) with respect to $y$ gives

$$
\frac{\mathrm{d}}{\mathrm{dt}}\left(\frac{\partial \tilde{\mathrm{x}}_{\mathrm{i}}(\mathrm{t}, \mathrm{y})}{\partial \mathrm{y}}\right)=\nabla \lambda_{\mathrm{i}}\left(\mathrm{u}\left(\mathrm{t}, \tilde{\mathrm{x}}_{\mathrm{i}}(\mathrm{t}, \mathrm{y})\right)\right) \frac{\partial \mathrm{u}}{\partial \mathrm{x}}\left(\mathrm{t}, \tilde{\mathrm{x}}_{\mathrm{i}}(\mathrm{t}, \mathrm{y})\right) \frac{\partial \tilde{\mathrm{x}}_{\mathrm{i}}(\mathrm{t}, \mathrm{y})}{\partial \mathrm{y}}
$$

Then, noting (2.6), it follows from (2.31) that

$$
\begin{aligned}
& \frac{d\left(e^{L t} q_{i}\right)}{d_{i} t}=\frac{d\left(e^{L t} w_{i}\right)}{d_{i} t} \frac{\partial \tilde{x}_{i}(t, y)}{\partial y}+e^{L t} w_{i} \frac{d}{d_{i} t}\left(\frac{\partial \tilde{x}_{i}(t, y)}{\partial y}\right)= \\
& \left(\sum_{j, k=1}^{n} e^{L t}\left[\gamma_{i j k}(u) w_{j} w_{k}+\tilde{\gamma}_{i j k}(u) w_{j} w_{k}\right]+e^{L t} w_{i} \nabla \lambda_{i}(u) u_{x}\right) \frac{\partial \tilde{x}_{i}(t, y)}{\partial y}
\end{aligned}
$$

Thus, from (2.4), (2.7) and (2.32), we immediately get (2.28)-(2.30). The proof of Lemma 2.4 is finished.

Similarly, noting (2.4), by (2.13) and (2.31), we have

Lemma 2.5. Let $p i(t ; x)$ be defined by $\mathrm{p}_{\mathrm{i}}\left(\mathrm{t}, \tilde{\mathrm{x}}_{\mathrm{i}}(\mathrm{t}, \mathrm{y})\right)=\mathrm{v}_{\mathrm{i}}\left(\mathrm{t}, \tilde{\mathrm{x}}_{\mathrm{i}}(\mathrm{t}, \mathrm{y})\right) \frac{\partial \tilde{\mathrm{x}}_{\mathrm{i}}(\mathrm{t}, \mathrm{y})}{\partial \mathrm{y}}$ Then along the ith

Characteristic $\mathrm{x}=\tilde{\mathrm{x}}_{\mathrm{i}}(\mathrm{t}, \mathrm{y})$ we have

$$
\frac{\mathrm{d}\left(\mathrm{e}^{\mathrm{Lt}} \mathrm{p}_{\mathrm{i}}\right)}{\mathrm{d}_{\mathrm{i}} \mathrm{t}}=\sum_{\mathrm{j}, \mathrm{k}=1}^{\mathrm{n}} \mathrm{e}^{\mathrm{Lt}} \mathrm{B}_{\mathrm{ijk}}(\mathrm{u}) \frac{\partial \tilde{x}_{\mathrm{i}}(\mathrm{t}, \mathrm{y})}{\partial \mathrm{y}} \mathrm{v}_{\mathrm{j}} \mathrm{w}_{\mathrm{k}}+\sum_{\mathrm{j}, \mathrm{k}=1}^{\mathrm{n}} \mathrm{e}^{\mathrm{L} t} \tilde{\beta}_{\mathrm{ijk}}(\mathrm{u}) \frac{\partial \tilde{\mathrm{x}}_{\mathrm{i}}(\mathrm{t}, \mathrm{y})}{\partial \mathrm{y}} \mathrm{v}_{\mathrm{j}} \mathrm{v}_{\mathrm{k}}
$$

Where $\tilde{\beta}_{\mathrm{ijk}}(\mathrm{u})$ is given by $(2.15)$ and

$\tilde{\beta}_{\mathrm{ijk}}(\mathrm{u})=\tilde{\beta}_{\mathrm{ijk}}(\mathrm{u})+\nabla \lambda_{\mathrm{i}}(\mathrm{u}) \mathrm{r}_{\mathrm{k}}(\mathrm{u}) \delta_{\mathrm{ij}}$

By (2.16), it is easy to see that

$$
\begin{array}{ll}
\mathrm{B}_{\mathrm{iji}}(\mathrm{u}) \equiv 0 & \forall \mathrm{i} \neq \mathrm{j}(\mathrm{i}, \mathrm{j}=1, \ldots, \mathrm{n}) \\
\mathrm{B}_{\mathrm{iji}}(\mathrm{u}) \equiv \nabla \lambda_{\mathrm{i}}(\mathrm{u}) \mathrm{r}_{\mathrm{i}}(\mathrm{u}) \quad \forall \mathrm{i}(\mathrm{i}=1, \ldots, \mathrm{n})
\end{array}
$$

Moreover, by (2.17), in the normalized coordinates we have

$$
\mathrm{B}_{\mathrm{iji}}\left(\mathrm{u}_{\mathrm{j}} \mathrm{e}_{\mathrm{j}}\right) \equiv 0 \quad \forall\left|\mathrm{uj}_{6}\right| \text { small, } \forall \mathrm{j} \neq \mathrm{i}
$$

while, when the ith characteristic $\lambda_{i}(u)$ is weakly linearly degenerate, in the normalized coordinates,

$$
\mathrm{B}_{\mathrm{iii}}\left(\mathrm{u}_{\mathrm{i}} \mathrm{e}_{\mathrm{i}}\right) \equiv 0 \quad \forall\left|\mathrm{u}_{\mathrm{i}}\right| \text { small } \forall \mathrm{i}
$$

Lemma 2.6. Let $z i(t ; x)$ be defined by $Z_{\mathrm{i}}\left(\mathrm{t}, \tilde{\mathrm{x}}_{\mathrm{i}}(\mathrm{t}, \mathrm{y})\right)=\mathrm{u}_{\mathrm{i}}\left(\mathrm{t}, \tilde{\mathrm{x}}_{\mathrm{i}}(\mathrm{t}, \mathrm{y})\right) \frac{\partial \tilde{\mathrm{x}}_{\mathrm{i}}(\mathrm{t}, \mathrm{y})}{\partial \mathrm{y}}$ Then along the ith characteristic

$\mathrm{x}=\tilde{\mathrm{x}}_{\mathrm{i}}(\mathrm{t}, \mathrm{y})$ we have

$\frac{\mathrm{d}\left(\mathrm{e}^{\mathrm{Lt}} \mathrm{Z}_{\mathrm{i}}\right)}{\mathrm{dit}}=\sum_{\mathrm{j}, \mathrm{k}=1}^{\mathrm{n}} \mathrm{e}^{\mathrm{Lt}} \mathrm{F}_{\mathrm{ijk}}(\mathrm{u}) \frac{\partial \tilde{\mathrm{x}}_{\mathrm{i}}(\mathrm{t}, \mathrm{y})}{\partial \mathrm{y}} \mathrm{u}_{\mathrm{j}} \mathrm{w}_{\mathrm{k}}$

Where

$\mathrm{F}_{\mathrm{ijk}}(\mathrm{u})=\mathrm{p}_{\mathrm{ijk}}(\mathrm{u})+\nabla \lambda_{\mathrm{i}}(\mathrm{u}) \mathrm{r}_{\mathrm{k}}(\mathrm{u}) \delta_{\mathrm{ij}}$

By (2.20) and (2.22), it is easy to see that

$$
\begin{array}{ll}
\mathrm{F}_{\mathrm{ijj}}(\mathrm{u}) \equiv 0, & \forall \mathrm{i} \neq \mathrm{j}(\mathrm{i}, \mathrm{j}=1, \ldots, \mathrm{n}) \\
\mathrm{F}_{\mathrm{iji}}(\mathrm{u}) \equiv 0, & \forall \mathrm{i} \neq \mathrm{j}(\mathrm{i}, \mathrm{j}=1, \ldots, \mathrm{n})
\end{array}
$$


And

$$
\mathrm{F}_{\mathrm{iji}}(\mathrm{u})=\nabla \lambda_{\mathrm{i}}(\mathrm{u}) \mathrm{r}_{\mathrm{i}}(\mathrm{u}) \quad \forall \mathrm{i}(\mathrm{i}=1, \ldots, \mathrm{n})
$$
gives

Proof. Differentiating the first equation of (2.27) with respect to $y$

$$
\frac{\mathrm{d}}{\mathrm{dt}}\left(\frac{\partial \tilde{\mathrm{x}}_{\mathrm{i}}(\mathrm{t}, \mathrm{y})}{\partial \mathrm{y}}\right)=\nabla \lambda_{\mathrm{i}}\left(\mathrm{u}\left(\mathrm{t}, \tilde{\mathrm{x}}_{\mathrm{i}}(\mathrm{t}, \mathrm{y})\right)\right) \frac{\partial \mathrm{u}}{\partial \mathrm{x}}\left(\mathrm{t}, \tilde{\mathrm{x}}_{\mathrm{i}},(\mathrm{t}, \mathrm{y})\right) \frac{\partial \tilde{\mathrm{x}}_{\mathrm{i}}(\mathrm{t}, \mathrm{y})}{\partial \mathrm{y}}
$$

Then, noting (2.19), it follows from (2.44) that

$$
\begin{aligned}
& \frac{d\left(e^{\mathrm{Lt}} \mathrm{Z}_{\mathrm{i}}\right)}{\mathrm{d}_{\mathrm{i}} \mathrm{t}}=\frac{\mathrm{d}\left(\mathrm{e}^{\mathrm{Lt}} \mathrm{Z}_{\mathrm{i}}\right)}{\mathrm{d}_{\mathrm{i}} \mathrm{t}} \frac{\partial \tilde{\mathrm{x}}_{\mathrm{i}}(\mathrm{t}, \mathrm{y})}{\partial \mathrm{y}}+\mathrm{e}^{\mathrm{Lt}} \mathrm{Z}_{\mathrm{i}} \frac{\mathrm{d}}{\mathrm{d}_{\mathrm{i}} \mathrm{t}}\left(\frac{\partial \tilde{x}_{\mathrm{i}}(\mathrm{t}, \mathrm{y})}{\partial \mathrm{y}}\right)= \\
& \left(\sum_{\mathrm{j}, \mathrm{k}=1}^{\mathrm{n}} \mathrm{e}^{\mathrm{Lt}} \mathrm{p}_{\mathrm{ijk}}(\mathrm{u}) \mathrm{u}_{\mathrm{j}} \mathrm{w}_{\mathrm{k}}+\mathrm{e}^{\mathrm{Lt}} \mathrm{Z}_{\mathrm{i}} \nabla \lambda_{\mathrm{i}}(\mathrm{u}) \mathrm{u}_{\mathrm{x}}\right) \frac{\partial \tilde{x}_{i}(\mathrm{t}, \mathrm{y})}{\partial \mathrm{y}}
\end{aligned}
$$

Thus, from (2.4), (2.20)-(2.22) and (2.45), we immediately get (2.39)-(2.43). The proof of Lemma 2.6 is finished.

\section{Proof of Theorem 1.1}

By the existence and uniqueness of a local $C 1$ solution for quasilinear hyperbolic systems [22], there exists $T 0>0$ such that the mixed initial-boundary value problem (1.1) and (1.12)-(1.13) admits a unique $C^{1}$ solution $u=u(t, x)$ on the domain

$$
\mathrm{D}\left(\mathrm{T}_{0}\right) \stackrel{\text { def }}{=}\left\{(\mathrm{t}, \mathrm{x}) \mid 0 \leq \mathrm{t} \leq \mathrm{T}_{\mathrm{o}}, \mathrm{x} \geq 0\right\}
$$

Thus, in order to prove Theorem 1.1 it suffices to establish a uniform a priori estimate for the $\mathrm{C}^{0}$ norm of $\mathrm{u}$ and $\mathrm{u}_{\mathrm{x}}$ on any given domain of existence of the $\mathrm{C}^{1}$ solution $\mathrm{u}=\mathrm{u}(\mathrm{t} ; \mathrm{x})$.

Noting (1.2) and (1.10), we have

$$
\lambda_{1}(0)<\ldots<\lambda_{\mathrm{m}}(0)<\lambda_{\mathrm{m}+1}(0)<\ldots<\lambda_{\mathrm{n}}(0)
$$

Thus, there exist sufficiently small positive constants $\delta$ and $\delta_{0}$ such that

$$
\begin{array}{ll}
\lambda_{\mathrm{i}+1}(\mathrm{u})-\lambda_{\mathrm{i}}(\mathrm{v}) \geq 4 \delta_{0} & \forall|\mathrm{u}|,|\mathrm{v}| \leq \delta(\mathrm{i}-1, \ldots, \mathrm{n}-1) \\
\lambda_{\mathrm{i}}(\mathrm{u})-\lambda_{\mathrm{i}}(\mathrm{v}) \leq \frac{\delta_{0}}{2} & \forall|\mathrm{u}|,|\mathrm{v}| \leq \delta(\mathrm{i}-1, \ldots, \mathrm{n})
\end{array}
$$

And

$$
\left|\lambda_{\mathrm{i}}(0)\right| \geq \delta_{0} \quad(\mathrm{i}=1, \ldots, \mathrm{n})
$$

For the time being it is supposed that on the domain of existence of the $C^{1}$ solution $u=u(t ; x)$ to the mixed initial-boundary value problem (1.1) and (1.12)-(1.13), we have

$$
|\mathrm{u}(\mathrm{t}, \mathrm{x})| \leq \delta
$$

At the end of the proof of Lemma 3.3, we will explain that this hypothesis is reasonable. Thus, in order to prove Theorem 1.1, we only need to establish a uniform a priori estimate for the piecewise $C^{0}$ norm of $v$ and $w$ defined by (1.14) and (2.1) on the domain of existence of the $C^{l}$ solution $u=u(t ; x)$.

For any fixed $T>0$, let

$$
\begin{aligned}
& \mathrm{D}_{+}^{\mathrm{T}}=\left\{(\mathrm{t}, \mathrm{x}) \mid 0 \leq \mathrm{t} \leq \mathrm{T}, \mathrm{x} \geq\left(\lambda_{\mathrm{n}}(0)+\delta_{0}\right) \mathrm{t}\right\} \\
& \mathrm{D}_{-}^{\mathrm{T}}=\left\{(\mathrm{t}, \mathrm{x}) \mid 0 \leq \mathrm{t} \leq \mathrm{T}, 0 \leq \mathrm{x} \leq\left(\lambda_{\mathrm{m}+1}(0)+\delta_{0}\right) \mathrm{t}\right\} \\
& \mathrm{D}^{\mathrm{T}}=\left\{(\mathrm{t}, \mathrm{x}) \mid 0 \leq \mathrm{t} \leq \mathrm{T},\left(\lambda_{\mathrm{m}+1}(0)-\delta_{0}\right) \mathrm{t} \leq \mathrm{x} \leq\left(\lambda_{\mathrm{n}}(0)+\delta_{0}\right) \mathrm{t}\right\}
\end{aligned}
$$

and for $i=m+1 ;::: ; n$; let

$$
\begin{aligned}
& \mathrm{D}^{\mathrm{T}}=\left\{(\mathrm{t}, \mathrm{x}) \mid 0 \leq \mathrm{t} \leq \mathrm{T},-\left[\delta_{0}+\mathrm{n}\left(\lambda_{\mathrm{i}}(0)-\lambda_{\mathrm{m}+1}(0)\right)\right] \mathrm{t}\right. \\
& \left.\leq \mathrm{x}-\lambda_{\mathrm{i}}(0) \mathrm{t} \leq\left[\delta_{0}+\mathrm{n}\left(\lambda_{\mathrm{n}}(0)\right)\right] \mathrm{t}\right\}
\end{aligned}
$$

where $n>0$ is suitably small (Figure 1 ).

Noting that $n>0$ is small, by (3.3), it is easy to see that

$\mathrm{D}_{\mathrm{i}}^{\mathrm{T}} \cap \mathrm{D}_{\mathrm{j}}^{\mathrm{T}}=\varnothing, \quad \forall_{\mathrm{i}} \neq \mathrm{j}$

And

$$
\bigcup_{i=m+1}^{n} D_{i}^{T} \subset D^{T}
$$

By the definitions of $D_{i}^{T}$ and $D^{T}$, it is easy to get the following lemma. Lemma 3.1. For each $i=m+1, \ldots, n$, on the domain $D^{T} / D_{i}^{T}$ we have

$$
\text { ct } \leq\left|\mathrm{x}-\lambda_{\mathrm{i}}(0) \mathrm{t}\right| \leq \mathrm{Ct}, \mathrm{cx} \leq\left|\mathrm{x}-\lambda_{\mathrm{i}}(0) \mathrm{t}\right| \leq \mathrm{Cx}
$$

where $c$ and $C$ are positive constants independent of $T$.

Let

$$
\begin{aligned}
& \mathrm{V}\left(\mathrm{D}_{+}^{\mathrm{T}}\right)=\max _{\mathrm{i}=1, \ldots, \mathrm{n}}\left\|(1+\mathrm{x})^{1+\mu}{ }_{\mathrm{vi}}(\mathrm{t}, \mathrm{x})\right\|_{\mathrm{L} \infty\left(\mathrm{D}_{+}^{\mathrm{T}}\right)} \\
& \mathrm{W}\left(\mathrm{D}_{+}^{\mathrm{T}}\right)=\max _{\mathrm{i}=1, \ldots, \mathrm{n}}\left\|(1+\mathrm{t})^{1+\mu}{ }_{\mathrm{wi}}(\mathrm{t}, \mathrm{x})\right\|_{\mathrm{L} \infty\left(\mathrm{D}_{+}^{\mathrm{T}}\right)} \\
& \mathrm{V}\left(\mathrm{D}_{-}^{\mathrm{T}}\right)=\max _{\mathrm{i}=1, \ldots, \mathrm{n}}\left\|(1+\mathrm{t})^{1+\mu}{ }_{\mathrm{wi}}(\mathrm{t}, \mathrm{x})\right\|_{\mathrm{L} \infty\left(\mathrm{D}_{-}^{\mathrm{T}}\right)} \\
& \mathrm{W}\left(\mathrm{D}_{-}^{\mathrm{T}}\right)=\max _{\mathrm{i}=1, \ldots, \mathrm{n}}\left\|(1+\mathrm{t})^{1+\mu}{ }_{w i}(\mathrm{t}, \mathrm{x})\right\|_{\mathrm{L}_{\infty}\left(\mathrm{D}_{-}^{\mathrm{T}}\right)} \\
& \mathrm{V}_{\infty}^{\mathrm{c}}(\mathrm{T})=\max \left\{\max _{\mathrm{r}=1, \ldots, \mathrm{m}} \sup _{(\mathrm{t}, \mathrm{x}) \in \mathrm{D}^{\mathrm{T}}}(1+\mathrm{T})^{1+\mu}\left|\mathrm{V}_{\mathrm{r}}(\mathrm{t}, \mathrm{x})\right|\right. \\
& \left.\max _{\mathrm{s}=\mathrm{m}+1, \ldots, \mathrm{n}, \mathrm{n}} \sup _{(\mathrm{t}, \mathrm{x}) \in \mathrm{D}^{\mathrm{T}} \backslash \mathrm{D}_{\mathrm{s}}^{\mathrm{T}}}\left(1+\left|\mathrm{x}-\lambda_{\mathrm{s}}(0) \mathrm{t}\right|\right)^{1+\mu}\left|\mathrm{v}_{\mathrm{s}}(\mathrm{t}, \mathrm{x})\right|\right\} \\
& \mathrm{W}_{\infty}^{\mathrm{c}}(\mathrm{T})=\max \left\{\max _{\mathrm{r}=1, \ldots, \mathrm{m}} \sup _{(\mathrm{t}, \mathrm{x}) \in \mathrm{D}^{\mathrm{T}}}(1+\mathrm{T})^{1+\mu}\left|\mathrm{W}_{\mathrm{r}}(\mathrm{t}, \mathrm{x})\right|\right. \\
& \left.\max _{\mathrm{s}=\mathrm{m}+1, \ldots, \mathrm{n}} \sup _{(\mathrm{t}, \mathrm{x}) \in \mathrm{D}^{\mathrm{T}} \backslash \mathrm{D}_{\mathrm{s}}^{\mathrm{T}}}\left(1+\left|\mathrm{x}-\lambda_{\mathrm{s}}(0) \mathrm{t}\right|\right)^{1+\mu}\left|\mathrm{W}_{\mathrm{s}}(\mathrm{t}, \mathrm{x})\right|\right\} \\
& \mathrm{U}_{\infty}^{\mathrm{c}}(\mathrm{T})=\max \left\{\max _{\mathrm{r}=1, \ldots, \mathrm{m}} \sup _{(\mathrm{t}, \mathrm{x}) \in \mathrm{D}^{\mathrm{T}}}(1+\mathrm{t})^{1+\mu}\left|\mathrm{u}_{\mathrm{r}}(\mathrm{t}, \mathrm{x})\right|\right. \\
& \left.\max _{\mathrm{s}=\mathrm{m}+1, \ldots, \mathrm{n}} \sup _{(\mathrm{t}, \mathrm{x}) \in \mathrm{D}^{\mathrm{T}} \backslash \mathrm{D}_{\mathrm{s}}^{\mathrm{T}}}\left(1+\left|\mathrm{x}-\lambda_{\mathrm{s}}(0) \mathrm{t}\right|\right)^{1+\mu}\left|\mathrm{W}_{\mathrm{s}}(\mathrm{t}, \mathrm{x})\right|\right\} \\
& \tilde{V}_{1}(T)=\max _{i=m+1, \ldots, n} \max _{j \neq 1} \sup _{\tilde{c}_{j}} \int_{\tilde{C}_{j}}\left|V_{i}(t, x)\right| d t
\end{aligned}
$$

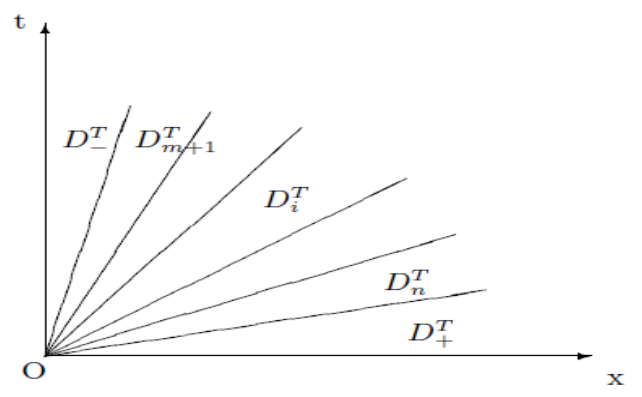

Figure 1: Where $n>0$ is suitably small. 


$$
\begin{aligned}
& \tilde{\mathrm{W}}_{1}(\mathrm{~T})=\max _{\mathrm{i}=\mathrm{m}+1, \ldots, \mathrm{n}} \max _{\mathrm{j} \neq 1} \sup _{\tilde{\mathrm{c}}_{\mathrm{j}}} \int_{\tilde{\mathrm{C}}_{\mathrm{j}}}\left|\mathrm{W}_{\mathrm{i}}(\mathrm{t}, \mathrm{x})\right| \mathrm{dt} \\
& \tilde{U}_{1}(\mathrm{~T})=\max _{\mathrm{i}=\mathrm{m}+1, \ldots, \mathrm{n}} \max _{\mathrm{j} \neq 1} \sup _{\tilde{\mathrm{c}}_{\mathrm{j}}} \int_{\tilde{\mathrm{C}}_{\mathrm{j}}}\left|\mathrm{U}_{\mathrm{i}}(\mathrm{t}, \mathrm{x})\right| \mathrm{dt}
\end{aligned}
$$

Where $\tilde{\mathrm{C}}_{\mathrm{j}}$ denotes any given $\mathrm{jth}$ characteristic in

$$
\begin{aligned}
& D_{i}^{T}(j \neq i, i=m+1, . ., n) \\
& V 1(T)=\max _{i=m+, . ., n} \sup _{0 \leq t \leq T} \int_{D_{i}^{T}(t)}\left|v_{i}(t, x)\right| d x \\
& W_{1}(T)=\max _{i=m+, ., n} \sup _{0 \leq t \leq T} \int_{D_{i}^{T}(t)}\left|w_{i}(t, x)\right| d x \\
& U_{1}(T)=\max _{i=m+, . ., n} \sup _{0 \leq t \leq T} \int_{D_{i}^{T}(t)}\left|u_{i}(t, x)\right| d x
\end{aligned}
$$

Where $D_{i}^{T}(t)(t \geq 0)$ denotes the t-section of $D_{i}^{T}$

$$
\begin{aligned}
& D_{i}^{T}(t)=\left\{(t, x) \mid T=t,(t, x) \in D_{i}^{T}\right\} \\
& V \propto(T)=\max _{i=1, \ldots, n} \sup _{0 \leq t \leq T \leq x \geq 0}\left|V_{i}(t, x)\right|
\end{aligned}
$$

And

$$
\mathrm{V}_{\infty}(\mathrm{T})=\max _{\mathrm{i}=1, \ldots, \mathrm{n}} \sup _{0 \leq \mathrm{t} \leq \mathrm{T} \leq \mathrm{x} \geq 0}\left|\mathrm{w}_{\mathrm{i}}(\mathrm{t}, \mathrm{x})\right|
$$

Clearly, $\mathrm{V}_{\infty}(\mathrm{T})$ is equivalent to

$$
\mathrm{U}_{\infty}(\mathrm{T})=\max _{\mathrm{i}=1, \ldots, \mathrm{n}} \sup _{0 \leq \mathrm{t} \leq \mathrm{T} \leq \mathrm{x} \geq 0}\left|\mathrm{U}_{\mathrm{i}}(\mathrm{t}, \mathrm{x})\right|
$$

In the present situation, similar to the corresponding result in [24,30-33], we have

Lemma 3.2. Suppose that in a neighborhood of $u=0 ; \mathrm{A}(\mathrm{u}) \in \mathrm{C}^{2}$ system (1.1) is strictly hyperbolic and (1.10) holds. Suppose furthermore that $\varphi(x)$ satisfies (1.15). Then there exists a sufficiently small $\theta_{0}>0$ such that for any fixed $\theta_{0} \in\left[0, \theta_{0}\right]$ on any given existence domain $\{(\mathrm{t}, \mathrm{x}) \mid 0 \leq \mathrm{t} \leq \mathrm{T}, \mathrm{x} \geq 0\}$ of the $C^{1}$ solution $u=u(t ; x)$ to the mixed initialboundary value problem (1.1) and (1.12)-(1.13), we have the following uniform a priori estimates:

$$
\mathrm{V}\left(\mathrm{D}_{+}^{\mathrm{T}}\right), \mathrm{W}\left(\mathrm{D}_{+}^{\mathrm{T}}\right) \leq \mathrm{k}_{1} \theta
$$

where here and henceforth, $k i(i=1 ; 2, \ldots$.$) are positive constants$ independent of $\theta$ and $T$.

Proof. We first estimate $\mathrm{W}\left(\mathrm{D}_{+}^{\mathrm{T}}\right)$

(i) For $i=1, \ldots, m$, let $\xi=\mathrm{x}_{\mathrm{i}}(\mathrm{s}, \mathrm{y})$ be the ith characteristic passing through any fixed point $(\mathrm{t}, \mathrm{x}) \in \mathrm{D}_{+}^{\mathrm{T}}$ and intersecting the $x$-axis at a point $(0 ; y)$. Noting (3.6), by (3.3)-(3.4), it is easy to see that the whole characteristic

$$
\xi=x_{i}(s, y)(0 \leq \mathrm{s} \leq \mathrm{t}) \text { is included in } \mathrm{D}_{+}^{\mathrm{T}}
$$

Noting (3.6), by (3.4) we get

$$
\mathrm{y}+\left(\lambda_{\mathrm{i}}(0)-\delta_{0} / 2\right) \mathrm{s} \leq \mathrm{x}_{\mathrm{i}}(\mathrm{s}, \mathrm{y}) \leq \mathrm{y} \quad \forall \mathrm{s} \in[\mathrm{o}, \mathrm{t}]
$$

By (3.4), it is easy to see that

$$
\mathrm{s} \leq \mathrm{t} \leq \mathrm{t}_{0}
$$

where $t 0$ denotes the $t$-coordinate of the intersection point of the straight line $\mathrm{x}=\left(\lambda_{\mathrm{n}}(0)+\delta_{0}\right) \mathrm{t}$ with the straight line $\mathrm{x}=\mathrm{y}+\left(\lambda_{\mathrm{i}}(0)+\delta_{0} / 2\right) \mathrm{t}$ passing through the point $(0 ; y)$. Clearly,

$$
\mathrm{t}_{0}=\frac{\mathrm{y}}{\lambda_{\mathrm{n}}(0)-\lambda_{\mathrm{i}}(0)+\delta_{0} / 2}
$$

Therefore it follows from (3.32)-(3.34) that

$$
\frac{\lambda_{\mathrm{n}}(0)}{\lambda_{\mathrm{n}}(0)-\lambda_{\mathrm{i}}(0)+\delta_{0} / 2} \mathrm{y} \leq \mathrm{x}_{\mathrm{i}}(\mathrm{s}, \mathrm{y}) \leq \mathrm{y}, \quad \forall \mathrm{s} \in[0, \mathrm{t}]
$$

By integrating (2.6) along this ith characteristic, we have

$$
\begin{aligned}
& \mathrm{w}_{\mathrm{i}}(\mathrm{t}, \mathrm{x})=\mathrm{e}^{-\mathrm{Lt}} \mathrm{w}_{\mathrm{i}}(\mathrm{o}, \mathrm{y})+\int_{0}^{\mathrm{t}} \mathrm{e}^{-\mathrm{L}(\mathrm{t}-\mathrm{s})}\left[\sum_{\mathrm{j}, \mathrm{k}=1}^{\mathrm{n}} \gamma_{\mathrm{ijk}}(\mathrm{u}) \mathrm{w}_{\mathrm{j}} \mathrm{w}_{\mathrm{k}}\right. \\
& \left.+\sum_{\mathrm{j}, \mathrm{k}=1}^{\mathrm{n}} \tilde{\gamma}_{\mathrm{ijk}}(\mathrm{u}) \mathrm{v}_{\mathrm{j}} \mathrm{w}_{\mathrm{k}}\right]\left(\mathrm{s}, \mathrm{x}_{\mathrm{i}}(\mathrm{s}, \mathrm{y})\right) \mathrm{ds}
\end{aligned}
$$

Thus, noting (3.6) and the fact that $L>0$, using(3.33)-(3.35), it follows from (3.36) that

$$
\begin{aligned}
(1+\mathrm{x})^{1+\mu}\left|\mathrm{w}_{\mathrm{i}}(\mathrm{t}, \mathrm{x})\right| \leq \mathrm{C}_{1}\left\{(1+\mathrm{y})^{1+\mu}\left|\mathrm{w}_{\mathrm{i}}(0, \mathrm{y})\right|+(1+\mathrm{y})^{-(1+\mu)} \mathrm{t}\left[\left(\mathrm{W}\left(\mathrm{D}_{+}^{\mathrm{T}}\right)\right)+\mathrm{W}\left(\mathrm{D}_{+}^{\mathrm{T}}\right) \mathrm{V}\left(\mathrm{D}_{+}^{\mathrm{T}}\right)\right]\right\} \\
\leq \mathrm{C}_{2}\left\{\theta+(1+\mathrm{y})^{-\mu} \mathrm{W}\left(\mathrm{D}_{+}^{\mathrm{T}}\right)\left[\mathrm{W}\left(\mathrm{D}_{+}^{\mathrm{T}}\right)+\mathrm{V}\left(\mathrm{D}_{+}^{\mathrm{T}}\right)\right]\right\} \\
\leq \mathrm{C}_{2}\left\{\theta+\mathrm{W}\left(\mathrm{D}_{+}^{\mathrm{T}}\right)\left[\mathrm{W}\left(\mathrm{D}_{+}^{\mathrm{T}}\right)+\mathrm{V}\left(\mathrm{D}_{+}^{\mathrm{T}}\right)\right]\right\}
\end{aligned}
$$

where here and henceforth, $C i(i=1 ; 2, \ldots$.$) will denote positive constants$ independent of and $T$.

(ii) For $i=m+1$; : : ; $n$; let $\xi=\mathrm{x}_{\mathrm{i}}(\mathrm{s}, \mathrm{y})$ be the ith characteristic passing through any fixed point $(\mathrm{t}, \mathrm{x}) \in \mathrm{D}_{+}^{\mathrm{T}}$ and intersecting the $x$-axis at a point $(0 ; y)$. Noting (3.6), by (3.3)-(3.4), it is easy to see that the whole characteristic $\xi=\mathrm{x}_{\mathrm{i}}(\mathrm{s}, \mathrm{y})(0 \leq \mathrm{s} \leq \mathrm{t})$ is included in $\mathrm{D}_{+}^{\mathrm{T}}$

Noting (3.6), by (3.4) we get

$\mathrm{y} \leq \mathrm{x}_{\mathrm{i}}(\mathrm{s}, \mathrm{y}) \leq \mathrm{y}+\lambda\left(\lambda_{\mathrm{i}}(0)+\delta_{0} / 2\right) \mathrm{s} \quad \forall \mathrm{s} \in[0, \mathrm{t}]$

By (3.4), it is easy to see that

$\mathrm{s} \leq \mathrm{t} \leq \mathrm{t}_{0}$

where $t 0$ denotes the $t$-coordinate of the intersection point of the straight line $\mathrm{x}=\left(\lambda_{\mathrm{n}}(0)+\delta_{0}\right) \mathrm{t}$ with the straight line $\mathrm{x}=\mathrm{y}+\left(\lambda_{\mathrm{i}}(0)+\delta_{0} / 2\right) \mathrm{t}$ passing through the point $(0 ; y)$. Clearly,

$$
\mathrm{t}_{0}=\frac{\mathrm{y}}{\lambda_{\mathrm{n}}(0)-\lambda_{\mathrm{i}}(0)+\delta_{0} / 2}
$$

Therefore it follows from (3.38)-(3.40) that

$\mathrm{y} \leq \mathrm{x}_{\mathrm{i}}(\mathrm{s}, \mathrm{y})=\frac{\lambda_{\mathrm{n}}(0)+\delta_{0}}{\lambda_{\mathrm{n}}(0)-\lambda_{\mathrm{i}}(0)+\delta_{0} / 2} \mathrm{y}, \forall \mathrm{s} \in[0, \mathrm{t}]$

Then, similar to (3.37), we have

$$
(1+\mathrm{x})^{1+\mu}\left|\mathrm{w}_{\mathrm{i}}(\mathrm{t}, \mathrm{x})\right| \leq \mathrm{C}_{3}\left\{\theta+\mathrm{w}\left(\mathrm{D}_{+}^{\mathrm{T}}\right)\left[\mathrm{W}\left(\mathrm{D}_{+}^{\mathrm{T}}\right)+\mathrm{V}\left(\mathrm{D}_{+}^{\mathrm{T}}\right)\right]\right\}
$$

Combining (3.37) and (3.42), we obtain

$$
\mathrm{W}\left(\mathrm{D}_{+}^{\mathrm{T}}\right) \leq \mathrm{C}_{4}\left\{\theta+\mathrm{W}\left(\mathrm{D}_{+}^{\mathrm{T}}\right)\left[\mathrm{W}\left(\mathrm{D}_{+}^{\mathrm{T}}\right)+\mathrm{V}\left(\mathrm{D}_{+}^{\mathrm{T}}\right)\right]\right\}
$$

Similarly, we have

$$
\mathrm{V}\left(\mathrm{D}_{+}^{\mathrm{T}}\right) \leq \mathrm{C}_{4}\left\{\theta+\mathrm{V}\left(\mathrm{D}_{+}^{\mathrm{T}}\right)\left[\mathrm{W}\left(\mathrm{D}_{+}^{\mathrm{T}}\right)+\mathrm{V}\left(\mathrm{D}_{+}^{\mathrm{T}}\right)\right]\right\}
$$

By (3.43) and (3.44), it is easy to prove that for $\mu>0$ suitably small, there exists a positive constant $k 1$ independent of $\theta$ and $T$, such that for any fixed $\mathrm{T}_{0}\left(0<\mathrm{T}_{0} \leq \mathrm{T}\right)$ if 
$\mathrm{W}\left(\mathrm{D}_{+}^{\mathrm{T}_{0}}\right), \mathrm{V}\left(\mathrm{D}_{+}^{\mathrm{T}_{0}}\right) \leq 2 \mathrm{k}_{1} \theta$

Then

$$
\mathrm{W}\left(\mathrm{D}_{+}^{\mathrm{T}_{0}}\right), \mathrm{V}\left(\mathrm{D}_{+}^{\mathrm{T}_{0}}\right) \leq \mathrm{k}_{1} \theta
$$

Hence, noting (1.15), by continuity we immediately get (3.31). The proof of Lemma 3.2 is finished. 2 Lemma 3.3. Under the assumptions of Lemma 3.2, suppose furthermore that system (1.1) is weakly linearly degenerate. Then in the normalized coordinates there exists a sufficiently small $\theta_{0}>0$ such that for any fixed $\theta \in\left[0, \theta_{0}\right]$ on any given existence domain $\{(\mathrm{t}, \mathrm{x}) \mid 0 \leq \mathrm{t} \leq \mathrm{T}, \mathrm{x} \geq 0\}$ of the $C 1$ solution $u=u(t ; x)$ to the mixed initial-boundary value problem (1.1) and (1.12)(1.13), we have the following uniform a priori estimates:

$$
\begin{aligned}
& \mathrm{W}\left(\mathrm{D}_{-}^{\mathrm{T}}\right) \leq \mathrm{k}_{2} \theta \\
& \mathrm{V}\left(\mathrm{D}_{-}^{\mathrm{T}}\right) \leq \mathrm{k}_{3} \theta \\
& \mathrm{U}_{\infty}^{\mathrm{c}}(\mathrm{T}) \leq \mathrm{k}_{4} \theta \\
& \mathrm{W}_{\infty}^{\mathrm{c}}(\mathrm{T}), \mathrm{V}_{\infty}^{\mathrm{c}}(\mathrm{T}) \leq \mathrm{k}_{5} \theta \\
& \tilde{\mathrm{W}}_{1}(\mathrm{~T}), \mathrm{W}_{1}(\mathrm{~T}), \tilde{\mathrm{V}}_{1}(\mathrm{~T}), \mathrm{V}_{1}(\mathrm{~T}), \tilde{\mathrm{U}}_{1}(\mathrm{~T}), \mathrm{U}_{1}(\mathrm{~T}) \leq \mathrm{k}_{6} \theta \\
& \mathrm{U}_{\infty}(\mathrm{T}), \mathrm{V}_{\infty}(\mathrm{T}) \leq \mathrm{k}_{7} \theta
\end{aligned}
$$

And

$$
\mathrm{W} \infty(\mathrm{T}) \leq \mathrm{k}_{8} \theta
$$

Proof. We first estimate $\mathrm{W}\left(\mathrm{D}_{-}^{\mathrm{T}}\right)$

For $j=1 ;::: ; m$, passing through any fixed point $(\mathrm{t}, \mathrm{x}) \in \mathrm{D}_{-}^{\mathrm{T}}$ we draw the jth characteristic $C_{j}: \xi=\xi_{j}(s, t, x)$ which must intersect the boundary $\mathrm{x}=\left(\lambda_{\mathrm{n}}(0)+\delta_{0}\right)$ t of $\mathrm{D}^{\mathrm{T}}$ at a point $(t 0 ; y)$. that

Proposition 3.1. On this jth characteristic $\mathrm{C}_{\mathrm{j}}: \xi=\xi_{\mathrm{j}}(\mathrm{s}, \mathrm{t}, \mathrm{x})$ it follows

$$
\mathrm{t} \geq \mathrm{t}_{0} \geq \frac{-\lambda_{\mathrm{j}}(0)-\frac{\delta_{0}}{2}}{\lambda_{\mathrm{n}}(0)-\lambda_{\mathrm{j}}(0)+\frac{\delta_{0}}{2}} \mathrm{t}
$$

Proof. Noting (3.4), it is easy to see that

$$
\mathrm{x}-\left(\lambda_{\mathrm{j}}(0)+\frac{\delta_{0}}{2}\right) \mathrm{t} \leq \mathrm{y}-\left(\lambda_{\mathrm{j}}(0)+\frac{\delta_{0}}{2}\right) \mathrm{t}_{0}
$$

On the other hand, from (3.8), we have

$$
\mathrm{x} \geq 0
$$

Since

$$
\mathrm{y}=\left(\lambda_{\mathrm{n}}(0)+\delta_{0}\right) \mathrm{t}_{0}
$$

we conclude from (3.55)-(3.57) that

$$
\mathrm{t}_{0} \geq \frac{-\lambda_{\mathrm{j}}(0)-\frac{\delta_{0}}{2}}{\lambda_{\mathrm{n}}(0) \lambda_{\mathrm{j}}-\frac{\delta_{0}}{2}} \mathrm{t}
$$

Noting the fact that $\mathrm{t} \geq \mathrm{t}_{0}$ we immediately get (3.54).

By integrating (2.6) along $\xi=\xi_{\mathrm{j}}(\mathrm{s} ; \mathrm{t}, \mathrm{x})$ and noting (2.9) and (2.11), we have

$$
\begin{aligned}
& \mathrm{w}_{\mathrm{j}}(\mathrm{t}, \mathrm{x})=\mathrm{e}-\mathrm{L}^{\left(\mathrm{t}-\mathrm{t}_{0}\right)} \mathrm{w}_{\mathrm{j}}\left(\mathrm{t}_{0}, \mathrm{y}\right)
\end{aligned}
$$

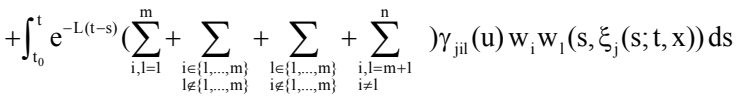

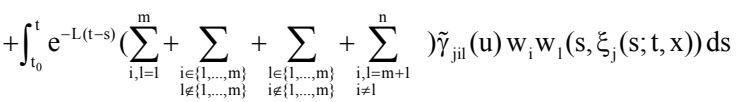

$$
\begin{aligned}
& \left.+\int_{t_{0}}^{t} e^{-L(t-s)}\left[\sum_{1=m+1}^{n} \tilde{\gamma}_{j i l}(u)-\tilde{\gamma}_{j i 1}\left(u_{1} e_{1}\right)\right) v_{1} w_{1}\right]\left(s, \xi_{j}(s ; t, x)\right) d s
\end{aligned}
$$

By using Lemma 3.2 and noting (3.54) and (3.57), it is easy to see that $\left|\mathrm{w}_{\mathrm{j}}\left(\mathrm{t}_{0}, \mathrm{y}\right)\right| \leq \mathrm{k}_{1} \theta(1+\mathrm{y})^{-(1+\mu)} \leq \mathrm{C}_{6} \theta\left(1+\mathrm{t}_{0}\right)^{-(1+\mu)} \leq \mathrm{C}_{7} \theta(1+\mathrm{t})^{-(1+\mu)}$

By Hadamard's formula, we have

$$
\tilde{\gamma}_{j l l}(u)-\tilde{\gamma}_{j l l}\left(u_{1} e_{1}\right)=\int_{0}^{1} \sum_{\substack{k=1 \\ k \neq 1}}^{n} \frac{\partial \tilde{\gamma}_{j l l}}{\partial u_{k}}\left(t_{1}, \ldots, T u_{1-1}, \ldots, T u_{n}\right) u_{k} d_{T}
$$

Thus, noting the fact that $L>0$, and using (3.13) and (3.54), we obtain from (3.59) that

$$
\begin{aligned}
& (1+\mathrm{t})^{1+\mu}\left|\mathrm{w}_{\mathrm{j}}(\mathrm{t}, \mathrm{x})\right| \leq \mathrm{C}_{8}\left\{\theta+\left(\mathrm{W}\left(\mathrm{D}_{-}^{\mathrm{T}}\right)\right)^{2}+\mathrm{W}\left(\mathrm{D}_{-}^{\mathrm{T}}\right) \mathrm{V}\left(\mathrm{D}_{-}^{\mathrm{T}}\right)\right. \\
& +\mathrm{W}_{\infty}^{\mathrm{c}}(\mathrm{T})\left[\tilde{\mathrm{W}}_{1}(\mathrm{~T})+\mathrm{W}_{\infty}^{\mathrm{c}}(\mathrm{T})+\tilde{\mathrm{V}}_{1}(\mathrm{~T})+\mathrm{V}_{\infty}^{\mathrm{c}}(\mathrm{T})+\tilde{\mathrm{U}}_{1}(\mathrm{~T}) \mathrm{V}_{\infty}(\mathrm{T})+\mathrm{U}_{\infty}^{\mathrm{c}}(\mathrm{T}) \mathrm{V}_{\infty}(\mathrm{T})\right] \\
& \left.+\tilde{\mathrm{W}}_{1}(\mathrm{~T})\left[\mathrm{V}_{\infty}^{\mathrm{c}}(\mathrm{T})+\mathrm{U}_{\infty}^{\mathrm{c}}(\mathrm{T}) \mathrm{V}_{\infty}(\mathrm{T})\right]\right\}
\end{aligned}
$$

Similar to Lemma 3.2 in [21], differentiating the nonlinear boundary condition (1.13) with respect to $t$, we get

$$
\begin{aligned}
& \mathrm{x}=0: \frac{\partial \mathrm{v}_{\mathrm{s}}}{\partial \mathrm{t}}=\sum_{\mathrm{r}=1}^{\mathrm{m}} \frac{\partial \mathrm{G}_{\mathrm{s}}}{\partial \mathrm{v}_{\mathrm{r}}}\left(\alpha(\mathrm{t}), \mathrm{v}_{1}, . ., \mathrm{v}_{\mathrm{m}}\right) \frac{\partial \mathrm{v}_{\mathrm{r}}}{\partial \mathrm{t}} \\
& +\sum_{\mathrm{i}=1}^{\mathrm{k}} \frac{\partial \mathrm{G}_{\mathrm{s}}}{\partial \alpha_{\mathrm{r}}}\left(\alpha(\mathrm{t}), \mathrm{v}_{1}, \ldots, \mathrm{v}_{\mathrm{m}}\right) \alpha_{\mathrm{i}}^{\prime}(\mathrm{t})+\mathrm{h}_{\mathrm{s}}^{\prime}(\mathrm{t})(\mathrm{s}=\mathrm{m}+1, \ldots, \mathrm{n})
\end{aligned}
$$

By (1.1), (1.3) and (2.4), it is easy to see that

$$
\frac{\partial v i}{\partial t}=\frac{\partial}{\partial t}\left(l_{i}(u) u\right)=-\lambda_{i}(u) w_{i}+\sum_{k=1}^{n} a_{i k}(u) w_{k}-L^{T} \nabla l_{i}(u) u(i=1, \ldots, n)
$$

Where

$$
\mathrm{a}_{\mathrm{ik}}(\mathrm{u})=-\lambda_{\mathrm{k}}(\mathrm{u}) \mathrm{r}_{\mathrm{k}}^{\mathrm{T}}(\mathrm{u}) \nabla 1_{\mathrm{i}}(\mathrm{u}) \mathrm{u}
$$

Therefore it follows from (3.63)-(3.65) that

$$
\mathrm{x}=0:\left(\mathrm{I}_{\mathrm{n}-\mathrm{m}}-\mathrm{B}_{1}(\mathrm{u})\right)\left(\begin{array}{c}
\mathrm{w}_{\mathrm{m}+1} \\
\vdots \\
\mathrm{w}_{\mathrm{n}}
\end{array}\right)=\mathrm{B}_{2}\left(\begin{array}{c}
\mathrm{w}_{1} \\
\vdots \\
\mathrm{w}_{\mathrm{m}}
\end{array}\right)-\overrightarrow{\mathrm{B}}_{3}
$$

where $B 1(u)$ is a matrix whose elements are all $C 1$ functions of $u$, which satisfy

In-m-B1(u) is invertible; for sufficiently small $|\mathrm{u}|$

$B 2$ is an $(n-m) \times m$ matrix independent of $w i(i=1, \ldots, n)$;

$$
\overrightarrow{\mathrm{B}}_{3}=\left(\frac{\sum_{\mathrm{i}=1}^{\mathrm{k}} \frac{\partial \mathrm{G}_{\mathrm{s}}}{\partial \alpha_{\mathrm{i}}}\left(\alpha(\mathrm{t}), \mathrm{v}_{1}, \ldots, \mathrm{v}_{\mathrm{m}}\right) \alpha_{\mathrm{i}}^{\prime}(\mathrm{t})+\mathrm{h}_{\mathrm{s}}^{\prime}(\mathrm{t})+\mathrm{F}_{\mathrm{s}}(\mathrm{t}, \mathrm{u}) \mathrm{u}}{\lambda_{\mathrm{s}}(\mathrm{u})}\right)_{\mathrm{m}+1 \leq \mathrm{s} \leq \mathrm{n}}
$$

in which $F s(s=m+1, \ldots, n)$ are continuous functions of $t$ and $u$.

Thus, noting (3.6), for $\partial>0$ small enough, by (3.66)-(3.68) we easily get 


$$
\begin{aligned}
& x=0: w s=\sum_{j=1}^{m} f_{s j}(t, u) w_{j}+\sum_{i=1}^{k} f_{s i}(t, u) \alpha_{i}^{\prime}(t) \\
& +\sum_{1=m+1}^{n} \bar{f}_{s l}(t, u) h_{1}^{\prime}(t)+f_{s}(t, u) u(s=m+1, \ldots, n)
\end{aligned}
$$

Where $\mathrm{f}_{\mathrm{s}}, \mathrm{f}_{\mathrm{sj}}, \overline{\mathrm{f}}_{\mathrm{si}}$ and $\overline{\mathrm{s}}_{\mathrm{sl}}$ are continuous functions of $t$ and $u$.

For $j=m+1 ;::: ; n$, passing through any fixed point $(\mathrm{t}, \mathrm{x}) \in \mathrm{D}_{-}^{\mathrm{T}}$ we draw the jth characteristic $c_{j}: \xi=\xi_{j}(\mathrm{~s} ; \mathrm{t}, \mathrm{x})$ which must intersect the $\mathrm{t}$-axis at a point $(t 0 ; 0)$. Then, we have that

Proposition 3.2. On this $j$ th characteristic $c_{j}: \xi=\xi_{j}(s ; t, x)$ it follows

$$
\mathrm{t} \geq \mathrm{t}_{0} \geq \frac{\lambda_{\mathrm{j}}(0)-\lambda_{\mathrm{m}+1}(0)+\frac{\delta_{0}}{2}}{\lambda_{\mathrm{j}}(0)-\frac{\delta_{0}}{2}} \mathrm{t}
$$

Proof. Noting (3.4), it is easy to see that

$\mathrm{x}-\left(\lambda_{\mathrm{j}}(0)-\frac{\delta_{0}}{2}\right) \mathrm{t} \geq-\left(\lambda_{\mathrm{j}}(0)-\frac{\delta_{0}}{2}\right) \mathrm{t}_{0}$

On the other hand, by (3.8), we have

$\mathrm{x} \leq\left(\lambda_{\mathrm{m}+1}(0)-\delta_{0}\right) \mathrm{t}$

Therefore, it follows from (3.71)-(3.72) that

$$
\mathrm{t}_{0} \geq \frac{\lambda_{\mathrm{j}}(0)-\lambda_{\mathrm{m}+1}(0)+\frac{\delta_{0}}{2}}{\lambda_{\mathrm{j}}(0)-\frac{\delta_{0}}{2}} \mathrm{t}
$$

Noting the fact that $\mathrm{t} \geq \mathrm{t}_{0}$ we immediately get (3.70).

By integrating (2.6) along $c_{j}: \xi=\xi_{j}(s ; t, x)$ we have

$$
\begin{aligned}
& \mathrm{w}_{\mathrm{j}}(\mathrm{t}, \mathrm{x})=\mathrm{e}^{-\mathrm{L}(\mathrm{t}-\mathrm{t} 0)} \mathrm{wj}\left(\mathrm{t}_{0}, 0\right)+\int_{\mathrm{t}_{0}}^{\mathrm{t}} \mathrm{e}^{-\mathrm{L}(\mathrm{t}-\mathrm{s})} \sum_{\mathrm{k}, \mathrm{l}=1}^{\mathrm{n}}\left[\gamma_{\mathrm{jkl}}(\mathrm{u}) \mathrm{w}_{\mathrm{k}} \mathrm{w}_{1}\right] \\
& \left.+\bar{\gamma}_{\mathrm{jkl}}(\mathrm{u}) \mathrm{v}_{\mathrm{k}} \mathrm{w}_{1}\right]\left(\mathrm{s}, \xi_{\mathrm{j}}(\mathrm{s} ; \mathrm{t}, \mathrm{x})\right) \mathrm{ds}
\end{aligned}
$$

By (3.69), we have

$$
\begin{aligned}
& \mathrm{w}_{\mathrm{j}}\left(\mathrm{t}_{0}, 0\right)=\sum_{\mathrm{r}=1}^{\mathrm{m}} \mathrm{f}_{\mathrm{jr}}\left(\mathrm{t}_{0}, \mathrm{u}\left(\mathrm{t}_{0}, 0\right)\right) \mathrm{w}_{\mathrm{r}}\left(\mathrm{t}_{0}, 0\right)+\sum_{\mathrm{i}=1}^{\mathrm{k}} \overline{\mathrm{f}}_{\mathrm{ji}}\left(\mathrm{t}_{0}, \mathrm{u}\left(\mathrm{t}_{0}, 0\right)\right) \alpha_{\mathrm{i}}^{\prime}\left(\mathrm{t}_{0}, 0\right) \\
& +\sum_{\mathrm{l}=\mathrm{m}+1}^{\mathrm{n}} \overline{\mathrm{f}}_{\mathrm{jl}}\left(\mathrm{t}_{0}, \mathrm{u}\left(\mathrm{t}_{0}, 0\right)\right) \mathrm{h}_{1}^{\prime}\left(\mathrm{t}_{0}\right)+\mathrm{f}_{\mathrm{j}}\left(\mathrm{t}_{0}, \mathrm{u}\left(\mathrm{t}_{0}, 0\right)\right) \mathrm{u}\left(\mathrm{t}_{0}, 0\right)
\end{aligned}
$$

By employing the same arguments as in (i), we can obtain

$$
\begin{aligned}
& \left(1+\mathrm{t}_{0}\right)^{1+\mu}\left|\operatorname{wr}\left(\mathrm{t}_{0}, 0\right)\right| \leq \mathrm{c}_{9}\left\{\theta+\left(\mathrm{W}\left(\mathrm{D}_{-}^{\mathrm{T}}\right)\right)^{2}+\mathrm{W}\left(\mathrm{D}_{-}^{\mathrm{T}}\right) \mathrm{V}\left(\mathrm{D}_{-}^{\mathrm{T}}\right)\right. \\
& +\mathrm{W}_{\infty}^{\mathrm{c}}(\mathrm{T})\left[\overline{\mathrm{W}}_{1}(\mathrm{~T})+\mathrm{W}_{\infty}^{\mathrm{c}}(\mathrm{T})+\overline{\mathrm{V}}_{1}(\mathrm{~T})+\mathrm{V}_{\infty}^{\mathrm{c}}(\mathrm{T})+\overline{\mathrm{U}}_{1}(\mathrm{~T}) \mathrm{V}_{\infty}(\mathrm{T})+\mathrm{U}_{\infty}^{\mathrm{c}}(\mathrm{T}) \mathrm{V}_{\infty}(\mathrm{T})\right] \\
& \left.+\overline{\mathrm{W}}_{1}(\mathrm{~T})\left[\mathrm{V}_{\infty}^{\mathrm{c}}(\mathrm{T})+\mathrm{U}_{\infty}^{\mathrm{c}}(\mathrm{T}) \mathrm{V}_{\infty}(\mathrm{T})\right]\right\}
\end{aligned}
$$

Thus, noting (1.15), (3.6) and (3.70), it follows from (3.75) and (3.76) that

$$
\begin{aligned}
& \left(1+\mathrm{t}_{0}\right)^{1+\mu}\left|\mathrm{w}_{\mathrm{j}}\left(\mathrm{t}_{0}, 0\right)\right| \leq \mathrm{c}_{10}\left(1+\mathrm{t}_{0}\right)^{1+\mu}\left|\mathrm{w}_{\mathrm{j}}\left(\mathrm{t}_{0}, 0\right)\right| \\
& \leq \mathrm{c}_{11}\left\{\theta+\left(\mathrm{V}\left(\mathrm{D}_{-}^{\mathrm{T}}\right)\right)^{2}+\mathrm{W}\left(\mathrm{D}_{-}^{\mathrm{T}}\right) \mathrm{W}\left(\mathrm{D}_{-}^{\mathrm{T}}\right)\right. \\
& +\mathrm{W}_{\infty}^{\mathrm{c}}(\mathrm{T})\left[\overline{\mathrm{W}}_{1}(\mathrm{~T})+\mathrm{W}_{\infty}^{\mathrm{c}}(\mathrm{T})+\overline{\mathrm{V}}_{1}(\mathrm{~T})+\mathrm{V}_{\infty}^{\mathrm{c}}(\mathrm{T})+\overline{\mathrm{U}}_{1}(\mathrm{~T}) \mathrm{V}_{\infty}(\mathrm{T})+\mathrm{U}_{\infty}^{\mathrm{c}}(\mathrm{T}) \mathrm{V}_{\infty}(\mathrm{T})\right] \\
& \left.+\overline{\mathrm{W}}_{1}(\mathrm{~T})\left[\mathrm{V}_{\infty}^{\mathrm{c}}(\mathrm{T})+\mathrm{U}_{\infty}^{\mathrm{c}}(\mathrm{T}) \mathrm{V}_{\infty}(\mathrm{T})\right]\right\}
\end{aligned}
$$

Hence, noting the fact that $L>0$, we obtain from (3.74) that $(1+t)^{1+\mu}|w j(t, x)| \leq C 12\left\{\theta+V\left(D_{-}^{\mathrm{T}}\right)+\left(\mathrm{W}\left(\mathrm{D}_{-}^{\mathrm{T}}\right)\right)^{2}+\mathrm{W}\left(\mathrm{D}_{-}^{\mathrm{T}}\right) \mathrm{V}\left(\mathrm{D}_{-}^{\mathrm{T}}\right)+\mathrm{W}_{\infty}^{\mathrm{c}}(\mathrm{T})\right.$

$\left[\bar{W}_{1}(T)+\mathrm{W}_{\infty}^{\mathrm{c}}(\mathrm{T})+\overline{\mathrm{V}}_{1}(\mathrm{~T})+\mathrm{V}_{\infty}^{\mathrm{c}}(\mathrm{T})+\overline{\mathrm{U}}_{1}(\mathrm{~T}) \mathrm{V}_{\infty}(\mathrm{T})+\mathrm{U}_{\infty}^{\mathrm{c}}(\mathrm{T}) \mathrm{V}_{\infty}(\mathrm{T})\right]$

$\left.+\overline{\mathrm{W}}_{1}(\mathrm{~T})\left[\mathrm{V}_{\infty}^{\mathrm{c}}(\mathrm{T})+\mathrm{U}_{\infty}^{\mathrm{c}}(\mathrm{T}) \mathrm{V}_{\infty}(\mathrm{T})\right]\right\}$

Combining (3.62) with (3.78), we get

$\mathrm{W}\left(\mathrm{D}_{-}^{\mathrm{T}}\right) \leq \mathrm{C}_{13}\left\{\theta+\mathrm{V}\left(\mathrm{D}_{-}^{\mathrm{T}}\right)+\left(\mathrm{W}\left(\mathrm{D}_{-}^{\mathrm{T}}\right)\right)^{2}+\mathrm{W}\left(\mathrm{D}_{-}^{\mathrm{T}}\right) \mathrm{V}\left(\mathrm{D}_{-}^{\mathrm{T}}\right)+\mathrm{W}_{\infty}^{\mathrm{c}}(\mathrm{T})\right.$

$\left[\overline{\mathrm{W}}_{1}(\mathrm{~T})+\mathrm{W}_{\infty}^{\mathrm{c}}(\mathrm{T})+\overline{\mathrm{V}}_{1}(\mathrm{~T})+\mathrm{V}_{\infty}^{\mathrm{c}}(\mathrm{T})+\overline{\mathrm{U}}_{1}(\mathrm{~T}) \mathrm{V}_{\infty}(\mathrm{T})+\mathrm{U}_{\infty}^{\mathrm{c}}(\mathrm{T}) \mathrm{V}_{\infty}(\mathrm{T})\right]$

$\left.+\overline{\mathrm{W}}_{1}(\mathrm{~T})\left[\mathrm{V}_{\infty}^{\mathrm{c}}(\mathrm{T})+\mathrm{U}_{\infty}^{\mathrm{c}}(\mathrm{T}) \mathrm{V}_{\infty}(\mathrm{T})\right]\right\}$

We next estimate $\overline{\mathrm{W}}_{1}(\mathrm{~T})$

Let

$\overline{\mathrm{C}}_{\mathrm{j}}: \mathrm{x}=\mathrm{x}_{\mathrm{j}}(\mathrm{t})\left(\mathrm{t}_{1} \leq \mathrm{t} \leq \mathrm{t}_{2}\right)$

be any given $j$ th characteristic in $D_{i}^{T}(j \neq i, i=m+1, \ldots, n)$ By (3.4), the whole ith characteristic $x=x i(t)$ passing through $O(0 ; 0)$ is included in $\mathrm{D}_{\mathrm{i}}^{\mathrm{T}}$ Let $(t 0 ; x j(t 0))$ be the intersection point of this characteristic with $\overline{\mathrm{C}}_{\mathrm{j}}$ Passing through any given point $(t ; x j(t))$ on $\overline{\mathrm{C}}_{\mathrm{j}}$ we draw the ith characteristic $\xi=\bar{x}_{\mathrm{i}}(\mathrm{s}, \mathrm{y})$ which intersects one of the boundaries of DT , say $\mathrm{x}=\left(\lambda \mathrm{n}(0)+\delta_{0}\right) \mathrm{t} \quad\left(\operatorname{resp} \cdot \mathrm{x}=\left(\lambda_{\mathrm{m}+1}(0)-\delta_{0}\right) \mathrm{t}\right)$ at a point $\mathrm{A}_{\mathrm{y}}\left(\mathrm{y} /\left(\lambda_{\mathrm{n}}(0)+\delta_{0}\right), \mathrm{y}\right) \quad\left(\right.$ resp. $\mathrm{B}_{\mathrm{y}}\left(\mathrm{y} /\left(\lambda_{\mathrm{m}+1}(0)-\delta_{0}\right) \mathrm{y}\right)$ if $\mathrm{t}_{0} \leq \mathrm{t} \leq \mathrm{t}_{2}\left(\right.$ resp. $\left.\mathrm{t}_{1} \leq \mathrm{t} \leq \mathrm{t}_{0}\right)$ Clearly, we have

$$
\tilde{x}_{i}(\mathrm{t}, \mathrm{y})=\mathrm{x}_{\mathrm{j}}(\mathrm{t})
$$

which gives a one-to-one correspondence $t=t(y)$ between the segment $\overline{O A_{y 2}} \mid\left(\operatorname{resp} . \overline{B_{y 1} O}\right)$ and $\tilde{C}_{j}\left(\mathrm{t}_{0} \leq \mathrm{t} \leq \mathrm{t}_{2}\right)\left(\operatorname{resp} \tilde{C}_{j}\left(\mathrm{t}_{1} \leq \mathrm{t} \leq \mathrm{t}_{0}\right)\right)$. Thus, the integral on $\tilde{C}_{j}$ with respect to $t$ can be reduced to the integral with respect to $y$. Differentiating (3.80) with respect to $t$ gives

$$
d t=\frac{1}{\lambda_{j}\left(\mathrm{u}\left(\mathrm{t}, \tilde{\mathrm{x}}_{\mathrm{i}}(\mathrm{t}, \mathrm{y})\right)\right)-\lambda_{\mathrm{i}}\left(\mathrm{u}\left(\mathrm{t}, \tilde{\mathrm{x}}_{\mathrm{i}}(\mathrm{t}, \mathrm{y})\right)\right)} \frac{\partial \tilde{x}_{i}(\mathrm{t}, \mathrm{y})}{\partial y} d y
$$

in which $t=t(y)$. Then, noting (3.3) and (3.6), it is easy to see that in order to estimate

$$
\begin{aligned}
\int_{\tilde{c}_{j}}\left|\mathrm{w}_{\mathrm{i}}(\mathrm{t}, \mathrm{x})\right| d t & =\int_{t_{1}}^{t_{0}}\left|\mathrm{w}_{\mathrm{i}}\left(\mathrm{t}, \mathrm{x}_{\mathrm{j}}(\mathrm{t})\right)\right| \mathrm{dt}+\int_{t_{0}}^{t_{2}}\left|\mathrm{w}_{\mathrm{i}}\left(\mathrm{t}, \mathrm{x}_{\mathrm{j}}(\mathrm{t})\right)\right| \mathrm{dt} \\
& =\int_{t_{1}}^{t_{0}}\left|\mathrm{w}_{\mathrm{i}}\left(\mathrm{t}, \tilde{\mathrm{x}}_{\mathrm{j}}(\mathrm{t})\right)\right| \mathrm{dt}+\int_{t_{0}}^{t_{2}}\left|\mathrm{w}_{\mathrm{i}}\left(\mathrm{t}, \tilde{\mathrm{x}}_{\mathrm{j}}(\mathrm{t})\right)\right| \mathrm{dt}
\end{aligned}
$$

it suffices to estimate

$$
\int_{0}^{y_{1}}\left|\mathrm{q}_{\mathrm{i}}\left(\mathrm{t}, \tilde{\mathrm{x}}_{\mathrm{i}}(\mathrm{t}, \mathrm{y})\right)\right|_{t=t(\mathrm{y})} \text { dy and } \int_{0}^{y_{2}}\left|\mathrm{q}_{\mathrm{i}}\left(\mathrm{t}, \tilde{\mathrm{x}}_{\mathrm{i}}(\mathrm{t}, \mathrm{y})\right)\right|_{t=t(\mathrm{y})} d y
$$

We now estimate $\int_{0}^{y_{2}}\left|\mathrm{q}_{\mathrm{i}}\left(\mathrm{t}, \tilde{\mathrm{x}}_{\mathrm{i}}(\mathrm{t}, \mathrm{y})\right)\right|_{t=t(\mathrm{y})} d y$

By integrating (2.28) along $\xi=\tilde{x}_{i}(\mathrm{~s}, \mathrm{y})$ and noting (2.30) and the fact that $\left.\tilde{x}_{i}\left(\mathrm{y} / \lambda_{\mathrm{n}}(0)+\delta_{0}\right), \mathrm{y}\right)=\mathrm{y}$ we obtain

$$
\begin{aligned}
& \left.q_{i}\left(\mathrm{t}, \tilde{\mathrm{x}}_{\mathrm{i}}(\mathrm{t}, \mathrm{y})\right)\right|_{\mathrm{t}=\mathrm{(y}(\mathrm{y})}=e^{-L\left(\mathrm{~L}(\mathrm{y})-\frac{y}{\lambda_{n}(0)+\delta_{0}}\right)} w_{i}\left(\frac{y}{\lambda n(0)+\delta_{0}}, \mathrm{y}\right)\left\{1-\frac{\lambda_{i}\left(\mathrm{u}\left(\mathrm{y} /\left(\lambda_{\mathrm{n}}(0)+\delta_{0}\right), \mathrm{y}\right)\right.}{\lambda_{n}(0)+\delta_{0}}\right\}
\end{aligned}
$$

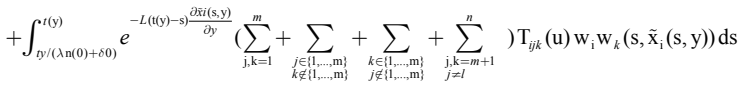

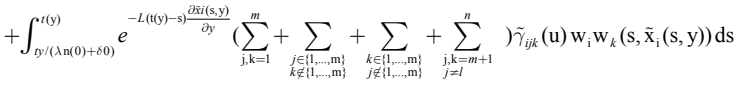

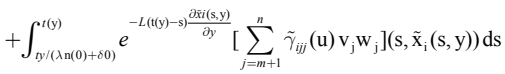

By Hadamard's formula and (2.11), we have 
Citation: Shao ZQ (2014) Global Classical Solutions to the Mixed Initial-boundary Value Problem for a Class of Quasilinear Hyperbolic Systems of Balance Laws. J Appl Computat Math 4: 197. doi:10.4172/2168-9679.1000197

Page 8 of 14

$\tilde{\gamma}_{i j j}(\mathrm{u})=\tilde{\gamma}_{i j j}(\mathrm{u})-\tilde{\gamma}_{i j j}\left(\mathrm{u}_{\mathrm{j}} \mathrm{e}_{\mathrm{j}}\right)=\int_{0}^{1} \sum_{l \neq j} \frac{\partial \tilde{\gamma}_{i j j}}{\partial u_{l}}\left(\mathrm{Tu}_{1}, \ldots, \mathrm{Tu}_{\mathrm{j}-1}, \mathrm{u}_{\mathrm{j}}, \mathrm{Tu}_{\mathrm{j}+1}, \ldots, \mathrm{Tu}_{\mathrm{n}}\right) \mathrm{u}_{1} \mathrm{~d}_{\mathrm{T}}$

Noting (3.6), (3.11), (3.13) and the fact that $L>0$ and $\frac{\tilde{\partial}_{x i}(\mathrm{~s}, \mathrm{y})}{\partial y}>0$ obtain from (3.84) and (3.85) that

$$
\begin{aligned}
& q_{i}\left(\mathrm{t}, \tilde{\mathrm{x}}_{\mathrm{i}}(\mathrm{t}, \mathrm{y})\right)|\mathrm{t}=\mathrm{t}(\mathrm{y}) \leq| \mathrm{w}_{\mathrm{i}}\left(\frac{y}{\lambda_{n}(0)+\partial_{0}} \mathrm{y}\right) \mid+\mathrm{C}_{15}\left\{\left[\left(\mathrm{~W}_{\infty}^{c}(\mathrm{~T})\right)^{2}+\mathrm{W}_{\infty}^{c}(\mathrm{~T}) \mathrm{V}_{\infty}^{c}(\mathrm{~T})+\mathrm{W}_{\infty}^{c}(\mathrm{~T}) \mathrm{U}_{\infty}^{c}(\mathrm{~T}) \mathrm{V}_{\infty}(\mathrm{T})\right]\right. \\
& \times \int_{y /(\lambda \mathrm{n}(0)+\delta 0)}^{t(\mathrm{y})}(1+\mathrm{s})^{-(1+\mu)}\left(1+\left|\tilde{\mathrm{x}}_{\mathrm{i}}(\mathrm{s}, \mathrm{y})\right|\right)^{-(1+\mu)} \frac{\partial \tilde{x}_{i}(\mathrm{~s}, \mathrm{y})}{\partial y} d s \\
& +\left[\mathrm{W}_{\infty}^{c}(\mathrm{~T})+V_{\infty}^{c}(\mathrm{~T})+U_{\infty}^{c}(\mathrm{~T}) \mathrm{V}_{\infty}(\mathrm{T})\right] \\
& \times \sum_{k=1}^{n} \int_{\left(\mathrm{s}, \tilde{x}_{i}(\mathrm{~s}, \mathrm{y})\right) \in \mathrm{D}_{l}^{\mathrm{f}}}(1+\mathrm{s})^{-(1-\mu)}\left|\mathrm{w}_{\mathrm{k}}\left(\mathrm{s}, \tilde{\mathrm{x}}_{\mathrm{i}}(\mathrm{s}, \mathrm{y})\right)\right| \frac{\partial \tilde{x}_{i}(\mathrm{~s}, \mathrm{y})}{\partial y} d s \\
& +W_{\infty}^{c}(\mathrm{~T}) \sum_{k=1}^{n} \int_{\left(\mathrm{s}, \tilde{\mathrm{x}}_{\mathrm{i}}(\mathrm{s}, \mathrm{y})\right) \in \mathrm{D}_{j}^{T}}(1+\mathrm{s})^{-(1-\mu)}\left|\mathrm{v}_{j}\left(\mathrm{~s}, \tilde{\mathrm{x}}_{\mathrm{i}}(\mathrm{s}, \mathrm{y})\right)\right| \frac{\partial \tilde{x}_{i}(\mathrm{~s}, \mathrm{y})}{\partial y} d s \\
& +W_{\infty}^{c}(\mathrm{~T}) \sum_{k=1}^{n} \int_{\left(\mathrm{s}, \tilde{\mathrm{x}}_{\mathrm{i}}(\mathrm{s}, \mathrm{y})\right) \in \mathrm{D}_{j}^{T}}(1+\mathrm{s})^{-(1-\mu)}\left|\mathrm{u}_{l}\left(\mathrm{~s}, \tilde{\mathrm{x}}_{\mathrm{i}}(\mathrm{s}, \mathrm{y})\right)\right| \frac{\partial \tilde{x}_{i}(\mathrm{~s}, \mathrm{y})}{\partial y} d s
\end{aligned}
$$

Noting that the transformation $\left\{\begin{array}{l}x=\tilde{x}_{i}(\mathrm{~s}, \mathrm{y}) \\ t=s\end{array}\right.$ gives the area element

$$
d t d x=\frac{\partial \tilde{x}_{i}(\mathrm{~s}, \mathrm{y})}{\partial y} d s d y
$$

by Lemma 3.2, it easily follows from (3.86) that

$$
\begin{aligned}
\int_{0}^{y_{2}}\left|\mathrm{q}_{\mathrm{i}}\left(\mathrm{t}, \tilde{\mathrm{x}}_{\mathrm{i}}(\mathrm{t}, \mathrm{y})\right)\right|_{t=t(y)} d y \leq C_{16}\left\{\theta+W_{\infty}^{c}(\mathrm{~T})\left[\mathrm{W}_{1}(\mathrm{~T})+W_{\infty}^{c}(\mathrm{~T})+W_{\infty}^{c}(\mathrm{~T})+V_{1}(\mathrm{~T})+V_{\infty}^{c}(\mathrm{~T})+\mathrm{U}_{1}(\mathrm{~T}) \mathrm{V}_{\infty}(\mathrm{T})\right]\right. \\
\left.\left.\left.+U_{\infty}^{c}(\mathrm{~T}) \mathrm{V}_{\infty}(\mathrm{T})\right]+\mathrm{W}_{1}(\mathrm{~T})\right]+\mathrm{W}_{1}(\mathrm{~T})\left[\mathrm{V}_{\infty}^{c}(\mathrm{~T})+V_{\infty}^{c}(\mathrm{~T})+U_{\infty}^{c}(\mathrm{~T}) \mathrm{V}_{\infty}(\mathrm{T})\right]\right]
\end{aligned}
$$

Similarly, we have

$\int_{0}^{y_{2}}\left|\mathrm{q}_{\mathrm{i}}\left(\mathrm{t}, \tilde{\mathrm{x}}_{\mathrm{i}}(\mathrm{t}, \mathrm{y})\right)\right|_{t=(\mathrm{y})} d y \leq C_{17}\left\{\theta+W_{\infty}^{c}(\mathrm{~T})\left[\mathrm{W}_{1}(\mathrm{~T})+W_{\infty}^{c}(\mathrm{~T})+W_{\infty}^{c}(\mathrm{~T})+V_{1}(\mathrm{~T})+V_{\infty}^{c}(\mathrm{~T})+\mathrm{U}_{1}(\mathrm{~T}) \mathrm{V}_{\infty}(\mathrm{T})\right]\right.$

Thus, we obtain

$\begin{aligned} \tilde{W}_{1}(\mathrm{~T}) \leq C_{17}\left\{\theta+W\left(\mathrm{D}_{-}^{T}\right)\right. & +\mathrm{W}_{\infty}^{c}(\mathrm{~T})\left[\mathrm{W}_{1}(\mathrm{~T})+W_{\infty}^{c}(\mathrm{~T})+W_{\infty}^{c}(\mathrm{~T})+V_{1}(\mathrm{~T})+V_{\infty}^{c}(\mathrm{~T})+\mathrm{U}_{1}(\mathrm{~T}) \mathrm{V}_{\infty}(\mathrm{T})\right] \\ & \left.\left.\left.+U_{\infty}^{c}(\mathrm{~T}) \mathrm{V}_{\infty}(\mathrm{T})\right]+\mathrm{W}_{1}(\mathrm{~T})\right]+\mathrm{W}_{1}(\mathrm{~T})\left[\mathrm{V}_{\propto}^{c}(\mathrm{~T})+U_{\infty}^{c}(\mathrm{~T})+\mathrm{V}_{\infty}(\mathrm{T})\right]\right]\end{aligned}$

We next estimate $W 1(T)$.

For $i=m+1, \ldots, n$, passing through any given point $(t ; x) \in D_{i}^{T}(\mathrm{t})$ we draw the ith characteristic $\xi=\tilde{x}_{i}(\mathrm{~s}, \mathrm{y})$ which intersects one of the boundaries of $D T$, say $x=\left(\lambda_{\mathrm{n}}(0)+\delta_{0}\right) \mathrm{t} \quad\left(\operatorname{resp} \cdot \mathrm{x}=\left(\lambda_{\mathrm{m}+1}(0)-\delta_{0}\right) \mathrm{t}\right.$ at a point $A_{y}\left(\mathrm{y} /\left(\lambda_{\mathrm{n}}(0)+\delta_{0}\right), \mathrm{y}\right)\left(\operatorname{resp} \cdot \mathrm{B}_{\mathrm{y}}\left(\mathrm{y} / \lambda_{\mathrm{m}+1}(0)-\delta_{0}\right) \mathrm{y}\right)$ Clearly, we have $x=\tilde{x}_{i}(\mathrm{t}, \mathrm{y})$ Therefore we obtain

$$
\int_{D_{i}^{T}(\mathrm{t})}\left|\mathrm{w}_{\mathrm{i}}(\mathrm{t}, \mathrm{x})\right| d x=\int_{0}^{y_{1}}\left|\mathrm{q}_{\mathrm{i}}\left(\mathrm{t}, \tilde{\mathrm{x}}_{\mathrm{i}}(\mathrm{t}, \mathrm{y})\right) \mathrm{dy}+\int_{0}^{y_{2}}\right| \mathrm{q}_{\mathrm{i}}\left(\mathrm{t}, \tilde{\mathrm{x}}_{\mathrm{i}}(\mathrm{t}, \mathrm{y})\right) \mathrm{dy}
$$

where $y 1$ and $y 2$ are shown in Figure 2.

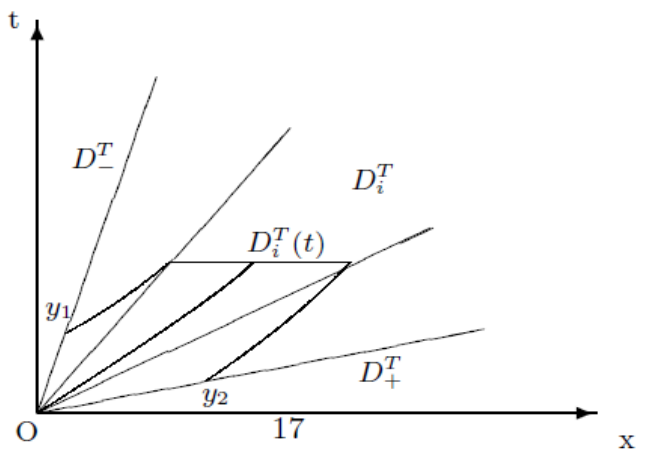

Figure 2: Where $y 1$ and $y 2$ are shown in figure.

$$
\begin{aligned}
& \text { Similar to (3.90), it follows from (3.91) that } \\
& \begin{aligned}
W_{1}(\mathrm{~T}) \leq C_{19}\left\{\theta+W\left(\mathrm{D}_{-}^{T}\right)+\right. & +\mathrm{W}_{\infty}^{c}(\mathrm{~T})\left[\mathrm{W}_{1}(\mathrm{~T})+W_{\infty}^{c}(\mathrm{~T})+W_{\infty}^{c}(\mathrm{~T})+V_{1}(\mathrm{~T})+V_{\infty}^{c}(\mathrm{~T})+\mathrm{U}_{1}(\mathrm{~T}) \mathrm{V}_{\infty}(\mathrm{T})\right] \\
& \left.\left.\left.+U_{\infty}^{c}(\mathrm{~T}) \mathrm{V}_{\infty}(\mathrm{T})\right]+\mathrm{W}_{1}(\mathrm{~T})\right]+\mathrm{W}_{1}(\mathrm{~T})\left[\mathrm{V}_{\infty}^{c}(\mathrm{~T})+U_{\infty}^{c}(\mathrm{~T})+\mathrm{V}_{\infty}(\mathrm{T})\right]\right]
\end{aligned}
\end{aligned}
$$

We next estimate $\mathrm{W}_{\infty}^{c}(\mathrm{~T})$

(i) For $r=1 ;::: ; m$, passing through any fixed point $(\mathrm{t}, \mathrm{x}) \in \mathrm{D}^{T}$ we draw the rth characteristic $C_{r}: \xi=\xi_{r}(\mathrm{~s} ; \mathrm{t}, \mathrm{x})$ which must intersect the boundary $x=\left(\lambda_{\mathrm{n}}(0)+\delta_{0}\right)$ t of $\mathrm{D}^{T}$ at a point $(t 0 ; y)$. Then, we have

Proposition 3.3. On this rth characteristic $C_{r}: \xi=\xi_{r}(\mathrm{~s} ; \mathrm{t}, \mathrm{x})$ it follows that

$$
t \geq t_{0} \geq \frac{\lambda_{m+1}(0)-\lambda_{\mathrm{r}}(0)-\frac{3 \delta_{0}}{2}}{\lambda_{n}(0)-\lambda_{\mathrm{r}}(0)-\frac{\delta_{0}}{2}} t
$$

Proof. By (3.4), it is easy to see that

$$
x-\left(\lambda_{\mathrm{r}}(0)+\frac{\delta_{0}}{2}\right) \mathrm{t} \leq \mathrm{y}-\left(\lambda_{\mathrm{r}}(0)+\frac{\delta_{0}}{2}\right) \mathrm{t}_{0}
$$

On the other hand, from (3.9), we have

$$
x \geq\left(\lambda_{\mathrm{m}+1}(0)-\delta_{0}\right) \mathrm{t}
$$

Since

$y=\left(\lambda_{\mathrm{n}}(0)+\delta_{0}\right) \mathrm{t}_{0}$

we conclude from (3.94)-(3.96) that

$$
t_{0} \geq \frac{\lambda_{m+1}(0)-\lambda_{\mathrm{r}}(0)-\frac{3 \delta_{0}}{2}}{\lambda_{n}(0)-\lambda_{\mathrm{r}}(0)-\frac{\delta_{0}}{2}} t
$$

Noting the fact that $t \geq t_{0}$ we immediately get (3.93).

By integrating (2.6) along $\xi=\xi_{r}(\mathrm{~s} ; \mathrm{t}, \mathrm{x})$ and noting (2.9) and (2.11), we have

$$
\begin{aligned}
& w_{r}(\mathrm{t}, \mathrm{x})=\mathrm{e}^{-\mathrm{L}\left(\mathrm{t}-\mathrm{t}_{0}\right)} \mathrm{W}_{\mathrm{r}}\left(\mathrm{t}_{0}, \mathrm{y}\right)
\end{aligned}
$$

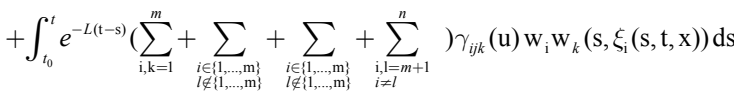

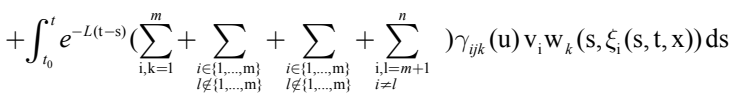

$$
\begin{aligned}
& \left.+\int_{t_{0}}^{t} e^{-L(\mathrm{t}-\mathrm{s})}\left[\sum_{l=m+1}^{n} \tilde{\gamma}_{r l l}(\mathrm{u})-\tilde{\gamma}_{r l l}\left(\mathrm{u}_{1} \mathrm{e}_{1}\right)\right) \mathrm{v}_{1} \mathrm{w}_{1}\right]\left(\mathrm{s}, \tilde{\mathrm{x}}_{\mathrm{i}}(\mathrm{s}, \mathrm{y})\right) \mathrm{ds}
\end{aligned}
$$

By using Lemma 3.2 and noting (3.93) and (3.96), it is easy to see that $\left|\mathrm{w}_{\mathrm{r}}\left(\mathrm{t}_{0}, \mathrm{y}\right)\right| \leq \mathrm{k}_{1} \theta(1+\mathrm{y})^{-(1+\mu)} \leq \mathrm{C}_{20} \theta(1+\mathrm{t})^{-(1+\mu)} \leq \mathrm{C}_{21} \theta(1+\mathrm{t})^{-(1+\mu)}$

By Hadamard's formula, we have

$$
\tilde{\gamma}_{r u}(u)-\tilde{\gamma}_{r u}\left(\mathrm{u}_{l} \mathrm{e}_{l}\right)=\int_{0}^{1} \sum_{k \neq l} \frac{\partial \tilde{\gamma}_{r} u}{\partial u_{k}}\left(\mathrm{TU}_{1}, . ., \mathrm{TU}_{l-1}, \mathrm{U}_{1}, T U_{l+1}, \ldots, T U_{n}\right) U_{k} d T .
$$

Thus, noting (3.93) and the fact that $L>0$, we obtain from (3.98) that

$$
\begin{gathered}
(1+\mathrm{t})^{1+\mu}\left|\mathrm{w}_{\mathrm{r}}(\mathrm{t}, \mathrm{x})\right| \leq \mathrm{C}_{22}\left\{\theta+W_{\infty}^{c}(\mathrm{~T})\left[\tilde{\mathrm{W}}_{1}(\mathrm{~T})+\mathrm{W}_{\infty}^{c}(\mathrm{~T})+\tilde{\mathrm{V}}_{1}(\mathrm{~T})+\mathrm{V}_{\infty}^{c}(\mathrm{~T})+\tilde{\mathrm{U}}_{1}(\mathrm{~T}) \mathrm{V}_{\infty}(\mathrm{T})\right.\right. \\
\left.\left.+\mathrm{U}_{\infty}^{c}(\mathrm{~T}) \mathrm{V}_{\infty}(\mathrm{T})\right]+\tilde{\mathrm{W}}_{1}(\mathrm{~T})\left[\mathrm{V}_{\infty}^{c}(\mathrm{~T})+U_{\infty}^{c}(\mathrm{~T}) \mathrm{V}_{\infty}(\mathrm{T})\right]\right\}
\end{gathered}
$$

(ii) For $i=m+1 ;::$; $n$, for any fixed point $(\mathrm{t}, \mathrm{x}) \in \mathrm{D}^{T}$ but $(\mathrm{t}, \mathrm{x}) \notin \mathrm{D}_{i}^{T}$ by the definition of $\mathrm{D}_{i}^{T}$ for fixing the idea we may assume that 
Citation: Shao ZQ (2014) Global Classical Solutions to the Mixed Initial-boundary Value Problem for a Class of Quasilinear Hyperbolic Systems of Balance Laws. J Appl Computat Math 4: 197. doi:10.4172/2168-9679.1000197

Page 9 of 14

$$
x-\lambda_{i}(0) t>\left[\delta_{0}+\eta\left(\lambda_{n}(0)-\lambda_{i}(0)\right)\right] t
$$

which implies $i<n$. Let $\xi=\xi_{i}(\mathrm{~s} ; \mathrm{t}, \mathrm{x})$ be the $i$ th characteristic passing through $(t ; x)$, which intersects the boundary $x=\left(\lambda_{\mathrm{n}}(0)+\delta_{0}\right) \mathrm{t}$ of $D^{T}$ at a point $(t 0 ; y)$ (Figure 3 ).

Recalling (3.4), it is easy to see that

$$
x-\left(\lambda_{i}(0)+\frac{\delta_{0}}{2}\right) t \leq y-\left(\lambda_{i}(0)+\frac{\delta_{0}}{2}\right) t_{0}
$$

Since

$$
y=\left(\lambda_{n}(0)+\delta_{0}\right) t_{0},
$$

recalling (3.102) and the fact that $t \geq t_{0}$ it follows from (3.103) that

$$
t \geq t_{0} \geq \eta t \text {. }
$$

By integrating (2.6) along $\xi=\xi_{i}(\mathrm{~s} ; \mathrm{t}, \mathrm{x})$ and noting (2.9) and (2.11), we have

$$
\begin{aligned}
& w_{j}(\mathrm{t}, \mathrm{x})=\mathrm{e}-\mathrm{L}^{\left(\mathrm{t}-\mathrm{t}_{0}\right)} \mathrm{w}_{\mathrm{j}}\left(\mathrm{t}_{0}, \mathrm{y}\right) \\
& +\int_{t_{0}}^{t} e^{-L(\mathrm{t}-\mathrm{s})}\left(\sum_{i, l=1}^{m}+\sum_{\substack{i \in\{1, \ldots, \mathrm{m}\} \\
\mid \notin\{1, \ldots, \mathrm{m}\}}}+\sum_{\substack{l \in\{1, \ldots, \mathrm{m}\} \\
\mathrm{i} \notin\{1, \ldots, \mathrm{m}\}}}+\sum_{\substack{i, l=m+1 \\
i \neq l}}^{n}\right) \gamma_{\mathrm{jil}}(\mathrm{u}) \mathrm{w}_{j} \mathrm{w}_{l}\left(\mathrm{~s}, \xi_{\mathrm{j}}(\mathrm{s} ; \mathrm{t}, \mathrm{x})\right) \mathrm{ds}
\end{aligned}
$$

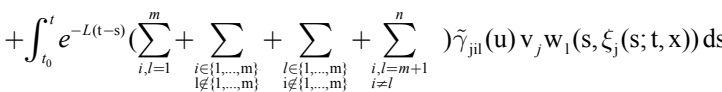

$$
\begin{aligned}
& \left.+\int_{t_{0}}^{t} e^{-L(\mathrm{t}-\mathrm{s})}\left[\sum_{l=m+1}^{n} \tilde{\gamma}_{\mathrm{jil}}(\mathrm{u})-\tilde{\gamma}_{\mathrm{jil}}\left(\mathrm{u}_{1} \mathrm{e}_{1}\right)\right) \mathrm{v}_{1} \mathrm{w}_{1}\right]\left(\mathrm{s}, \xi_{\mathrm{j}}(\mathrm{s} ; \mathrm{t}, \mathrm{x})\right) \mathrm{ds} \\
& +\int_{t_{0}}^{t} e^{-L(\mathrm{t}-\mathrm{s})} \tilde{\gamma}_{\mathrm{jil}}(\mathrm{u}) \mathrm{w}_{j} \mathrm{w}_{l}\left(\mathrm{~s}, \xi_{\mathrm{j}}(\mathrm{s} ; \mathrm{t}, \mathrm{x})\right) \mathrm{ds}
\end{aligned}
$$

By Lemma 3.2 and observing (3.104)-(3.105), it is easy to see that $\left|w_{i}\left(t_{0}, y\right)\right| \leq k_{1} \theta(1+y)^{-(1+\mu)} \leq C_{23} \theta\left(1+t_{0}\right)^{-(1+\mu)} \leq C_{24} \theta(1+t)^{-(1+\mu)}(3.107)$

By Hadamard's formula, we have

$$
\tilde{\gamma}_{i l l}(\mathrm{u})-\tilde{\gamma}_{\text {ill }}\left(u_{1} e_{1}\right)=\int_{0}^{1} \sum_{k \neq 1} \frac{\partial \tilde{\gamma}_{i l l}}{\partial u_{k}}\left(\mathrm{Tu}_{1}, \ldots, \mathrm{Tu}_{l-1}, u_{l}, T u_{l+1}, \ldots, T u_{n}\right) u_{k} d T
$$

Thus, recalling (3.13) and (3.105), and noting the fact that $L>0$, it follows from (3.106) that

$$
\begin{aligned}
& \left(1+\left|x-\lambda_{i}(0) \mathrm{t}\right|\right)^{1+\mu}\left|w_{i}(t, x)\right| \leq C_{25}\left\{\theta+W_{\infty}^{c}(T)\left[\tilde{W}(T)+W_{\infty}^{c}(T)+\tilde{\mathrm{V}}_{1}(T)+V_{\infty}^{c}(T)+\tilde{U}_{1}(\mathrm{~T}) \mathrm{V}_{\infty}(T)\right.\right. \\
& \left.\left.+\mathrm{U}_{\infty}^{c}(T) V_{\infty}(T)\right]+\tilde{W}_{1}(T)\left[V_{\infty}^{c}(T)+U_{\infty}^{c}(\mathrm{~T}) \mathrm{V}_{\infty}(T)\right]\right\}
\end{aligned}
$$

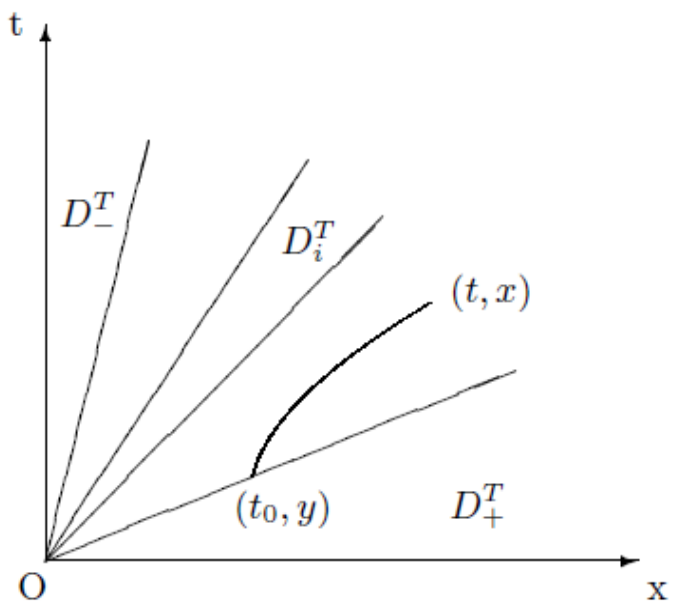

Figure 3: At a point $(t 0 ; y)$.
Next, we assume that

$$
x-\lambda_{i}(0) t<-\left[\delta_{0}+\eta\left(\lambda_{i}(0)-\lambda_{m+1}(0)\right)\right] t
$$

which implies $i>m+1$. Let $\xi=\xi_{i}(\mathrm{~s} ; \mathrm{t}, \mathrm{x})(t ; x)$, which intersects the boundary $x=\left(\lambda_{\mathrm{m}+1}(0)-\delta_{0}\right)$ t of $\mathrm{D}^{T}$ at a point $(t 0 ; y)$.

Recalling (3.4), it is easy to see that

$x-\left(\lambda_{i}(0)-\frac{\delta_{0}}{2}\right) t \geq y-\left(\lambda_{i}(0)-\frac{\delta_{0}}{2}\right) t_{0}$

Since

$y=\left(\lambda_{m+1}(0)-\delta_{0}\right) t_{0}$

noting (3.110) and the fact that $t \geq t_{0} n t$ it follows from (3.111) that

$$
t \geq t_{0} \geq \eta t
$$

By integrating (2.6) along $\xi=\xi_{i}(\mathrm{~s} ; \mathrm{t}, \mathrm{x})$ and noting (2.9) and (2.11), we have

$$
\begin{aligned}
& w_{i}(t, x)=e^{-L\left(t-t_{0}\right)} w_{i}\left(t_{0}, y\right)
\end{aligned}
$$

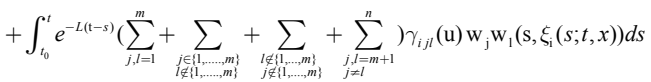

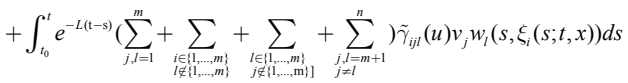

$$
\begin{aligned}
& +\int_{t_{0}}^{t} e^{-L(t-s)}\left[\sum_{l=m+1}^{n}\left(\tilde{\gamma}_{i l l}(u)-\tilde{\gamma}_{i l l}\left(u_{l} e_{l}\right)\right) v_{l} w_{l}\right]\left(s, \xi_{i}(s ; t, x)\right) d s \\
& +\int_{t_{0}}^{t} e^{-L(t-\mathrm{s})} \gamma_{i i i}(u) w_{i} w_{i}\left(s, \xi_{i}(s ; t, x)\right) d s
\end{aligned}
$$

By (3.113), it is easy to see that

$$
\left|w_{i}\left(t_{0}, y\right)\right| \leq C_{26} W\left(D_{-}^{T}\right)\left(1+t_{0}\right)^{-(1+\mu)} \leq C_{27} W\left(D_{-}^{T}\right)(1+t)^{-(1+\mu)}
$$

Thus, using (3.13), (3.108) and (3.113), and noting the fact that $L>$ 0 , it follows from (3.114) that

$$
\begin{aligned}
\left(1+\left.\left|x-\lambda_{i}(0) t\right|\right|^{1+\mu}\left|w_{i}(t, x)\right|\right. & \leq \\
& C_{28}\left\{W\left(D_{-}^{T}\right)+W_{\infty}^{c}(T)\left[\tilde{\mathrm{W}}_{1}(T)+W_{\infty}^{c}(T)+\tilde{\mathrm{V}}_{1}(\mathrm{~T})+\mathrm{V}_{\infty}^{c}(\mathrm{~T})\right.\right. \\
& \left.+\tilde{\mathrm{U}}_{1}(T) V_{\infty}(T)+U_{\infty}^{c}(T) V_{\infty}(T)\right]+\tilde{W}_{1}(T)\left[V_{\infty}^{c}(T)\right. \\
& \left.\left.+U_{\infty}^{c}(\mathrm{~T}) \mathrm{V}_{\infty}(T)\right]\right\}
\end{aligned}
$$

Combining (3.101) and (3.109), (3.116), we obtain

$$
\begin{aligned}
W_{\infty}^{c}(T) \leq & C_{29}\left\{\theta+W\left(D_{-}^{T}\right)+W_{\infty}^{c}(T)\left[\tilde{W}_{1}(T)+W_{\infty}^{c}(T)+\tilde{V}_{1}(\mathrm{~T})\right.\right. \\
& \left.+V_{\infty}^{c}(T)+\tilde{U}_{1}(T) V_{\infty}(T)+U_{\infty}^{c}(\mathrm{~T}) \mathrm{V}_{\infty}(T)\right]+\tilde{W}_{1}(T) \\
& {\left.\left[V_{\infty}^{c}(\mathrm{~T})+\mathrm{U}_{\infty}^{c}(T) \mathrm{V}_{\infty}(T)\right]\right\} }
\end{aligned}
$$

We next estimate $V\left(\mathrm{D}_{-}^{T}\right)$ (i) For $j=1 ;::: ; m$, for any fixed point $(\mathrm{t}, \mathrm{x}) \in \mathrm{D}_{-}^{T}$ similar to (3.59), by integrating (2.13) along $\xi=\xi_{i}(\mathrm{~s} ; \mathrm{t}, \mathrm{x})$ and noting (2.17)-(2.18), we have

$$
\begin{aligned}
& \mathrm{v}_{\mathrm{j}}(\mathrm{t}, \mathrm{x})=\mathrm{e}^{-\mathrm{L}\left(\mathrm{t}_{\mathrm{o}}\right)} \mathrm{v}_{\mathrm{j}}\left(\mathrm{t}_{0}, \mathrm{y}\right) \\
& +\int_{t_{0}}^{t} e^{-L(t-s)}\left(\sum_{\substack{i, l=1 \\
t_{0}}}^{m}+\sum_{\substack{i \in 1, \ldots, m\} \\
l \notin i, \ldots, m\}}}+\sum_{\substack{l \in\{1,1, m\} \\
i \notin\{1, \ldots, m\}}}+\sum_{\substack{l, l=m+1 \\
i \neq 1}}^{n}\right) \beta_{j i l}(u) v_{i} w_{l}\left(s, \xi_{j}(s ; t, x)\right) d s
\end{aligned}
$$

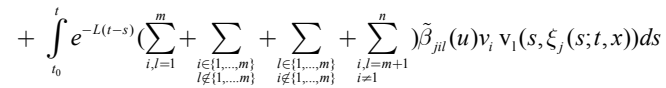

$$
\begin{aligned}
& +\int_{t_{0}}^{t} e^{-L(t-s)}\left[\sum_{l=m+1}^{n}\left(\beta_{j l}(u)-\beta_{j l}\left(u_{1} e_{1}\right)\right) v_{l} w_{l}\right]\left(s, \xi_{j}(s ; t, x)\right) d s \\
& +\int_{t_{0}}^{t} e^{-L(t-s)}\left[\sum_{l=m+1}^{n}\left(\tilde{\beta}_{j l l}(u)-\tilde{\beta}_{j l l}\left(u_{1} e_{1}\right)\right) v_{l}^{2}\right]\left(s, \xi_{j}(s ; t, x)\right) d s
\end{aligned}
$$

By using Lemma 3.2 and noting (3.54) and (3.57), it is easy to see that 
$\left|v_{j}\left(t_{0}, y\right)\right| \leq k_{1} \theta(1+y)^{-(1+\mu)} \leq C_{30} \theta(1+t 0)^{-(1+\mu)} \leq C_{31} \theta(1+t)^{-(1+\mu)}(3.119)$

By Hadamard's formula, we have

$\beta_{j l l}(u)-\beta_{j l l}\left(u_{l} e_{l}\right)=\int_{0}^{1} \sum_{\substack{k=1 \\ k \neq 1}}^{n} \frac{\partial \beta_{j l l}}{\partial u_{k}}\left(\mathrm{Tu}_{1}, \ldots, \mathrm{Tu}_{1-1}, \mathrm{u}_{1}, \mathrm{Tu}_{1+1}, \ldots ., \mathrm{Tu}_{\mathrm{n}}\right) \mathrm{u}_{\mathrm{k}} \mathrm{dT}(3.120)$

And

$\tilde{\beta}_{j l l}(u)-\tilde{\beta}_{j l l}\left(u_{l} e_{l}\right)=\int_{0}^{1} \sum_{\substack{k=1 \\ k \neq 1}}^{n} \frac{\partial \tilde{\beta}_{j l l}}{\partial u_{k}}\left(\mathrm{Tu}_{1}, \ldots . ., \mathrm{Tu}_{1-1}, \mathrm{u}_{1}, \mathrm{Tu}_{1+1}, \ldots ., \mathrm{Tu}_{\mathrm{n}}\right) \mathrm{u}_{\mathrm{k}} \mathrm{dT}$

Thus, noting the fact that $L>0$, and using (3.13) and (3.54), we obtain from (3.118) that

$$
\begin{aligned}
& (1+t)^{1+\mu}\left|v_{j}(t, x)\right| \leq \mathrm{C}_{32}\left\{\theta+\left(\mathrm{V}\left(\mathrm{D}_{-}^{T}\right)\right)^{2}+W\left(D_{-}^{T}\right) V\left(D_{-}^{T}\right)\right. \\
& +V_{\infty}^{c}(T)\left[\tilde{W}_{1}(T)+W_{\infty}^{c}(T)+\tilde{V}_{1}(T)+V_{\infty}^{c}(T)+\tilde{U}_{1}(T) V_{\infty}(T)+U_{\infty}^{c}(\mathrm{~T}) \mathrm{V}_{\infty}(T)\right] \\
& \left.+U_{\infty}^{c}(T) V_{\infty}(T)\left[W_{\infty}^{c}(T)+\tilde{W}_{1}(T)+\tilde{V}_{1}(T)\right]+W_{\infty}^{c}(T)\left[\tilde{V}_{1}(T)+\tilde{U}_{1}(T) V_{\infty}(\mathrm{T})\right]\right\}
\end{aligned}
$$

For $j=m+1 ;::: ; n$, for any fixed point $(t ; x) \in D_{-}^{T}$ similar to (3.74), we have

$$
\begin{aligned}
& v_{j}(t, x)=e^{-L\left(t-t_{0}\right)} v_{j}\left(t_{0}, 0\right)+\int_{t_{0}}^{t} e^{-L(t-s)} \sum_{k, l=1}^{n}\left[\beta_{j k l}(u) v_{k} w_{l}\right. \\
& \left.+\tilde{\beta}_{j k l}(\mathrm{u}) \mathrm{v}_{\mathrm{k}} \mathrm{v}_{1}\right]\left(\mathrm{s}, \xi_{\mathrm{j}}(\mathrm{s} ; \mathrm{t}, \mathrm{x})\right) \mathrm{ds}
\end{aligned}
$$

Noting (1.16), by (1.13), it is easy to see that

$$
v_{j}\left(t_{0}, 0\right)=\sum_{r=1}^{m} g_{j r}\left(t_{0}\right) v_{r}\left(t_{0}, 0\right)+h_{j}\left(t_{0}\right)
$$

Where

$$
g_{j r}\left(t_{0}\right)=\int_{0}^{1} \frac{\partial G_{j}}{\partial v_{r}}\left(\alpha\left(\mathrm{t}_{0}\right), \mathrm{Tv}_{1}\left(\mathrm{t}_{0}, 0\right), \ldots ., \mathrm{tv}_{\mathrm{m}}\left(\mathrm{t}_{0}, 0\right)\right) \mathrm{dT}
$$

By employing the same arguments as in (i), we can obtain

$$
\begin{aligned}
& \left(1+t_{0}\right)^{1+\mu}\left|v_{r}\left(t_{0}, 0\right)\right| \leq C_{33}\left\{\theta+\left(\mathrm{V}\left(\mathrm{D}_{-}^{T}\right)\right)^{2}+W\left(D_{-}^{T}\right) V\left(D_{-}^{T}\right)\right. \\
& +V_{\infty}^{c}(T)\left[\tilde{W}_{1}(T)+W_{\infty}^{c}(T)+\tilde{V}_{1}(T)+V_{\infty}^{c}(T)+\tilde{U}_{1}(T) V_{\infty}(T)+U_{\infty}^{c}(T) V_{\infty}(T)\right] \\
& \left.+\mathrm{U}_{\infty}^{c}(T) V_{\infty}(T)\left[W_{\infty}^{c}(T)+\tilde{W}_{1}(T)+\tilde{V}_{1}(T)\right]+W_{\infty}^{c}(T)\left[\tilde{V}_{1}(T)+\tilde{U}_{1}(T) V_{\infty}(T)\right]\right\}
\end{aligned}
$$

Thus, noting (1.15), (3.6) and (3.70), it follows from (3.124)-(3.126) that

$(1+t)^{1+\mu}\left|v_{j}\left(t_{0}, 0\right)\right| \leq C_{34}\left(1+t_{0}\right)^{1+\mu}\left|v_{j}\left(t_{0}, 0\right)\right| \leq C_{35}\{\theta$

$+\left(V\left(D_{-}^{T}\right)\right)^{2}+W\left(D_{-}^{T}\right) V\left(D_{-}^{T}\right)+V_{\infty}^{c}(T)\left[\tilde{W}_{1}(T)+W_{\infty}^{c}(T)+\tilde{V}_{1}(T)+V_{\infty}^{c}(T)\right.$

$\left.+\tilde{U}_{1}(T) V_{\infty}(T)+U_{\infty}^{c}(T) V_{\infty}(T)\right]+U_{\infty}^{c}(T) V_{\infty}(T)\left[W_{\infty}^{c}(\mathrm{~T})+\tilde{\mathrm{W}}_{1}(T)+\tilde{V}_{1}(T)\right]+$

$\left.W_{\infty}^{c}(T)\left[\tilde{V}_{1}(T)+\tilde{\mathrm{U}}_{1}(T) V_{\infty}(T)\right]\right\}$

Hence, noting the fact that $L>0$, we obtain from (3.123) that

$(1+t)^{1+\mu}\left|v_{j}(t, \mathbf{x})\right| \leq C_{36}\left\{\theta+\left(V\left(D_{-}^{T}\right)\right)^{2}+W\left(D_{-}^{T}\right) V\left(D_{-}^{T}\right)+V_{\infty}^{c}(T)\left[\tilde{W}_{1}(T)+W_{\infty}^{c}(T)+\tilde{V}_{1}(T)+V_{\infty}^{c}(T)\right.\right.$

$\left.+\tilde{U}_{1}(T) V_{\infty}(T)+U_{\infty}^{c}(T) V_{\infty}(T)\right]+U_{\infty}^{c}(T) V_{\infty}(T)\left[W_{\infty}^{c}(\mathrm{~T})+\tilde{\mathrm{W}}_{1}(T)+\tilde{V}_{1}(T)\right]+$

$\left.W_{\infty}^{c}(T)\left[\tilde{V}_{1}(T)+\tilde{\mathrm{U}}_{1}(T) V_{\infty}(T)\right]\right\}$

Combining (3.122) and (3.128), we get

$V\left(D_{-}^{T}\right) \leq C_{3\}}\left\{\theta+\left(V\left(D_{-}^{T}\right)\right)^{2}+W\left(D_{-}^{T}\right) V\left(D_{-}^{T}\right)+V_{\infty}^{c}(T)\left[\tilde{\mathrm{W}}_{1}(T)+W_{\infty}^{c}(T)+\tilde{V}_{1}(T)+V_{\infty}^{c}(T)\right.\right.$

$\left.+\tilde{U}_{1}(T) V_{\infty}(T)+U_{\infty}^{c}(T) V_{\infty}(T)\right]+U_{\infty}^{c}(T) V_{\infty}(T)\left[W_{\infty}^{c}(T)+\tilde{W}_{1}(T)+\tilde{V}_{1}(T)\right]+W_{\infty}^{c}(\mathrm{~T})\left[\tilde{V}_{1}(T)+(3.129)\right.$

$\left.\left.\tilde{U}_{1}(T) V_{\infty}(T)\right]\right\}$

We next estimate $\tilde{V}_{1}(\mathrm{~T})$ and $V 1(T)$.

For $i=m+1, \ldots, n$, for any given jth characteristic $\tilde{C}_{j}$ in $D_{i}^{T}(\mathrm{j} \neq \mathrm{i})$ as in the proof of (3.90), in order to estimate $\tilde{V}_{1}(\mathrm{~T})$ it suffices to estimate $\int_{0}^{y 1}\left|p_{i}\left(t, \tilde{x}_{i}(t, y)\right)\right|_{t=t(y)} d y$ and $\int_{0}^{y 2}\left|p_{i}\left(t, \tilde{x}_{i}(t, y)\right)\right|_{t=t(y)} d y$

By integrating (2.33) along $\xi=\tilde{x}_{i}(\mathrm{~s}, \mathrm{y})$ similar to (3.84), we have

$\left.P_{i}\left(\mathrm{t}, \tilde{\mathrm{x}}_{\mathrm{i}}(\mathrm{t}, \mathrm{y})\right)\right|_{\mathrm{t}=\mathrm{t}(\mathrm{y})}=e^{-\mathrm{L}\left(\mathrm{u}(\mathrm{y})-\frac{\mathrm{y}}{\left.\lambda_{n}(0)+\hat{\delta}_{0}\right)}\right)} v_{i}\left(\frac{y}{\lambda_{n}(0)+\delta_{0}}, \mathrm{y}\right)\left\{1-\frac{\lambda_{i}\left(\mathrm{u}\left(\mathrm{y} /\left(\lambda_{n}(0)+\delta_{0}\right), \mathrm{y}\right)\right.}{\lambda_{n}(0)+\delta_{0}}\right\}$

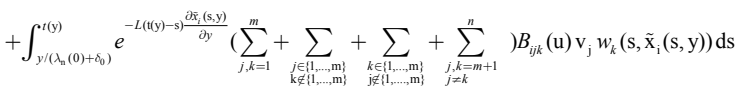

$+\int_{\left.y / \alpha_{0}(0)+\varepsilon_{0}\right)}^{t(\mathrm{y})} e-L(\mathrm{t}(\mathrm{y})-\mathrm{s}) \frac{\partial \tilde{x}_{i}(\mathrm{~s}, \mathrm{y})}{\partial y}\left[\sum_{j=m+1}^{n} B_{i j}(\mathrm{u}) \mathrm{v}_{\mathrm{j}} \mathrm{w}_{\mathrm{j}}\right]\left(\mathrm{s}, \tilde{\mathrm{x}}_{\mathrm{i}}(\mathrm{s}, \mathrm{y})\right) d s$

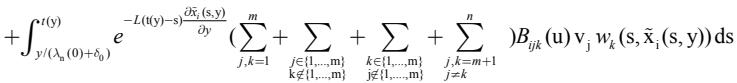

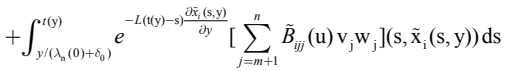

Noting that $\lambda_{i}(u)(i=m+1 ;:: ; n)$ are weakly linearly degenerate, by (2.37) and (2.38), we have

$B_{i j j}\left(\mathrm{u}_{\mathrm{j}} \mathrm{e}_{\mathrm{j}}\right) \equiv 0, \forall \mathrm{j}$

By Hadamard's formula, and noting (2.18) and (3.132), we have

$\operatorname{Bijj}(u)=B i j j(u) ; B i j j(u j e j)$

$=\int_{0}^{1} \sum_{l \neq j} \frac{\partial B_{i j j}}{\partial u_{l}}\left(T \mathrm{u}_{1}, \ldots, \mathrm{Tu}_{\mathrm{j}-1}, \mathrm{u}_{\mathrm{j}}, \mathrm{Tu}_{\mathrm{j}+1}, \ldots, \mathrm{T} \mathrm{u}_{\mathrm{n}}\right) u_{1} d_{T}$

And

$$
\begin{aligned}
\tilde{\beta}_{i j j}(\mathrm{u}) & =\tilde{\beta}_{i j j}(\mathrm{u})-\tilde{\beta}_{i j j}\left(\mathrm{u}_{\mathrm{j}} \mathrm{e}_{\mathrm{j}}\right) \\
& =\int_{0}^{1} \sum_{l \neq j} \frac{\partial \tilde{\beta}_{i j j}}{\partial u_{l}}\left(\mathrm{Tu}_{1}, \ldots ., \mathrm{Tu}_{\mathrm{j}-1}, \mathrm{uj}, \mathrm{Tu}_{\mathrm{j}+1}, \ldots, \mathrm{Tu}_{\mathrm{n}}\right) u_{\mathrm{l}} d_{T}
\end{aligned}
$$

Then, using Lemma 3.2, similar to (3.88), it follows from (3.131) that

$\int_{0}^{y 2}\left|\mathrm{p}_{\mathrm{i}}\left(\mathrm{t}, \tilde{\mathrm{x}}_{\mathrm{i}}(\mathrm{t}, \mathrm{y})\right)\right|_{t=t(\mathrm{y})} d y \leq C_{38}\left\{\theta+\mathrm{W}_{\infty}^{c}(\mathrm{~T})\left[\mathrm{V}_{1}(\mathrm{~T})+\mathrm{U}_{1}(\mathrm{~T}) \mathrm{V}_{\infty}(\mathrm{T})\right]\right.$

$+\mathrm{V}_{\infty}^{c}(\mathrm{~T})\left[\mathrm{W}_{1}(\mathrm{~T})+\mathrm{W}_{\infty}^{c}(\mathrm{~T})+\mathrm{V}_{1}(\mathrm{~T})+\mathrm{V}_{\infty}^{c}(\mathrm{~T})+\mathrm{U}_{1}(\mathrm{~T}) \mathrm{V}_{\infty}(\mathrm{T})+\mathrm{U}_{\infty}^{c}(\mathrm{~T}) \mathrm{V}_{\infty}(\mathrm{T})\right](3.135)$

$\left.+\mathrm{U}_{\infty}^{c}(\mathrm{~T}) \mathrm{V}_{\infty}(\mathrm{T})\left[\mathrm{W}_{\infty}^{c}(\mathrm{~T})+\mathrm{W}_{1}(\mathrm{~T})+\mathrm{V}_{1}(\mathrm{~T})\right]\right\}$

Similarly, we have

$\int_{0}^{y^{2}}\left|\mathrm{p}_{\mathrm{i}}\left(\mathrm{t}, \tilde{\mathrm{x}}_{\mathrm{i}}(\mathrm{t}, \mathrm{y})\right)\right|_{t=t(\mathrm{y})} d y \leq C_{39}\left\{\mathrm{~V}\left(\mathrm{D}_{-}^{T}\right)+\mathrm{W}_{\infty}^{c}(\mathrm{~T})\left[\mathrm{V}_{1}(\mathrm{~T})+\mathrm{U}_{1}(\mathrm{~T}) \mathrm{V}_{\infty}(\mathrm{T})\right]\right.$

$+\mathrm{V}_{\infty}^{c}(\mathrm{~T})\left[\mathrm{W}_{1}(\mathrm{~T})+\mathrm{W}_{\infty}^{c}(\mathrm{~T})+\mathrm{V}_{1}(\mathrm{~T})+\mathrm{V}_{\infty}^{c}(\mathrm{~T})+\mathrm{U}_{1}(\mathrm{~T}) \mathrm{V}_{\infty}(\mathrm{T})+\mathrm{U}_{\infty}^{c}(\mathrm{~T}) \mathrm{V}_{\infty}(\mathrm{T})\right](3.136)$

$\left.+\mathrm{U}_{\infty}^{c}(\mathrm{~T}) \mathrm{V}_{\infty}(\mathrm{T})\left[\mathrm{W}_{\infty}^{c}(\mathrm{~T})+\mathrm{W}_{1}(\mathrm{~T})+\mathrm{V}_{1}(\mathrm{~T})\right]\right\}$

Thus, we obtain

$\tilde{V}_{1} \leq C_{40}\left\{\mathrm{~V}\left(\mathrm{D}_{-}^{T}\right)+\mathrm{W}_{\infty}^{c}(\mathrm{~T})\left[\mathrm{V}_{1}(\mathrm{~T})+\mathrm{U}_{1}(\mathrm{~T}) \mathrm{V}_{\infty}(\mathrm{T})\right]\right.$

$+\mathrm{V}_{\infty}^{c}(\mathrm{~T})\left[\mathrm{W}_{1}(\mathrm{~T})+\mathrm{W}_{\infty}^{c}(\mathrm{~T})+\mathrm{V}_{1}(\mathrm{~T})+\mathrm{V}_{\infty}^{c}(\mathrm{~T})+\mathrm{U}_{1}(\mathrm{~T}) \mathrm{V}_{\infty}(\mathrm{T})+\mathrm{U}_{\infty}^{c}(\mathrm{~T}) \mathrm{V}_{\infty}(\mathrm{T})\right](3.137)$

$\left.+\mathrm{U}_{\infty}^{c}(\mathrm{~T}) \mathrm{V}_{\infty}(\mathrm{T})\left[\mathrm{W}_{\infty}^{c}(\mathrm{~T})+\mathrm{W}_{1}(\mathrm{~T})+\mathrm{V}_{1}(\mathrm{~T})\right]\right\}$

Similarly, we have

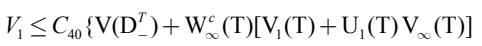

$+\mathrm{V}_{\infty}^{c}(\mathrm{~T})\left[\mathrm{W}_{1}(\mathrm{~T})+\mathrm{W}_{\infty}^{c}(\mathrm{~T})+\mathrm{V}_{1}(\mathrm{~T})+\mathrm{V}_{\infty}^{c}(\mathrm{~T})+\mathrm{U}_{1}(\mathrm{~T}) \mathrm{V}_{\infty}(\mathrm{T})+\mathrm{U}_{\infty}^{c}(\mathrm{~T}) \mathrm{V}_{\infty}(\mathrm{T})\right](3.138)$

$\left.+\mathrm{U}_{\infty}^{c}(\mathrm{~T}) \mathrm{V}_{\infty}(\mathrm{T})\left[\mathrm{W}_{\infty}^{c}(\mathrm{~T})+\mathrm{W}_{1}(\mathrm{~T})+\mathrm{V}_{1}(\mathrm{~T})\right]\right\}$

We next estimate $V_{\infty}^{c}(\mathrm{~T})$

(i) For $r=1 ;::: ; m$, for any fixed point $(t ; x) \in D T$, noting $(2.17)$ and (2.18), similar to (3.98), we

Have 


$$
\begin{aligned}
& V r(\mathrm{t}, \mathrm{x})=\mathrm{e}^{-\mathrm{L}\left(\mathrm{t}-\mathrm{t}_{0}\right)} V r\left(\mathrm{t}_{0}, \mathrm{y}\right)
\end{aligned}
$$

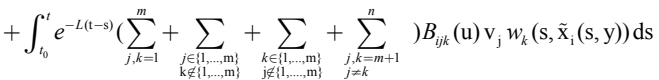

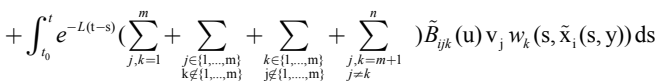

$$
\begin{aligned}
& +\int_{t_{0}}^{t} e^{-L(t-s)}\left[\sum_{l=m+1}^{n}\left(\tilde{\beta}_{\mathrm{rll}}(\mathrm{u})-\tilde{\beta}_{\mathrm{rll}}\left(\mathrm{u}_{1} \mathrm{e}_{1}\right)\right) \mathrm{v}_{l}^{2}\right](\mathrm{s}, \xi \mathrm{r}(\mathrm{s} ; \mathrm{t}, \mathrm{x})) \mathrm{ds} \\
& +\int_{t_{0}}^{t} e^{-L(t-s)}\left[\sum_{l=m+1}^{n}\left(\tilde{\beta}_{\mathrm{rll}}(\mathrm{u})-\tilde{\beta}_{\mathrm{rll}}\left(\mathrm{u}_{1} \mathrm{e}_{1}\right)\right) \mathrm{v}_{l}^{2}\right](\mathrm{s}, \xi \mathrm{r}(\mathrm{s} ; \mathrm{t}, \mathrm{x})) \mathrm{ds}
\end{aligned}
$$

By using Lemma 3.2 and noting (3.93) and (3.96), it is easy to see that $\left|\operatorname{Vr}\left(\mathrm{t}_{0}, \mathrm{y}\right)\right| \leq \mathrm{K}_{1} \theta(1+\mathrm{y})^{-(1+\mu)} \leq C_{42} \theta\left(1+\mathrm{t}_{0}\right)^{-(1+\mu)} \leq C_{43} \theta(1+\mathrm{t})^{-(1+\mu)}(3.140)$

By Hadamard's formula, we have

$$
\beta_{r l l}(\mathrm{u})-\beta_{r l l}\left(\mathrm{u}_{1} \mathrm{e}_{1}\right)=\int_{0}^{1} \sum_{k \neq l} \frac{\partial \beta_{r l l}}{\partial u_{k}}\left(\mathrm{Tu}_{1}, \ldots, \mathrm{Tu}_{1-1}, \mathrm{u}_{1}, \mathrm{Tu}_{1+1}, \ldots, \mathrm{Tu}_{\mathrm{n}}\right) u_{k} d_{T}
$$

And

$$
\tilde{\beta}_{r l l}(\mathrm{u})-\tilde{\beta}_{r l l}\left(\mathrm{u}_{1} \mathrm{e}_{1}\right)=\int_{0}^{1} \sum_{k \neq l} \frac{\partial \tilde{\beta}_{r l l}}{\partial u_{k}}\left(\mathrm{Tu}_{1}, \ldots, \mathrm{Tu}_{1-1}, \mathrm{u}_{1}, \mathrm{Tu}_{1+1}, \ldots ., \mathrm{Tu}_{\mathrm{n}}\right) u_{k} d_{T}
$$

Thus, noting (3.93) and the fact that $L>0$, we obtain from (3.139) that

$$
(1+\mathrm{t})^{1+\mu}\left|\mathrm{v}_{\mathrm{r}}(\mathrm{t}, \mathrm{x})\right| \leq C_{44}\left\{\theta+\mathrm{W}_{\infty}^{c}(\mathrm{~T})\left[\tilde{\mathrm{V}}_{1}(\mathrm{~T})+\tilde{\mathrm{U}}_{1}(\mathrm{~T}) \mathrm{V}_{\infty}(\mathrm{T})\right]\right.
$$

$+\mathrm{V}_{\infty}^{c}(\mathrm{~T})\left[\tilde{\mathrm{W}}_{1}(\mathrm{~T})+\mathrm{W}_{\infty}^{c}(\mathrm{~T})+\tilde{\mathrm{V}}_{1}(\mathrm{~T})+\mathrm{V}_{\infty}^{c}(\mathrm{~T})+\tilde{\mathrm{U}}_{1}(\mathrm{~T}) \mathrm{V}_{\infty}(\mathrm{T})+\mathrm{U}_{\infty}^{c}(\mathrm{~T}) \mathrm{V}_{\infty}(\mathrm{T})\right](3.143)$ $\left.+\mathrm{U}_{\infty}^{c}(\mathrm{~T}) \mathrm{V}_{\infty}(\mathrm{T})\left[\mathrm{W}_{\infty}^{c}(\mathrm{~T})+\tilde{\mathrm{W}}_{1}(\mathrm{~T})+\tilde{\mathrm{V}}_{1}(\mathrm{~T})\right]\right\}$

(ii) For $i=m+1 ;::: ; n$, for any fixed point $(\mathrm{t}, \mathrm{x}) \in \mathrm{D}^{\mathrm{T}}$ but $(\mathrm{t}, \mathrm{x}) \notin \mathrm{D}_{i}^{T}$ similar to (3.116), we have

$\left(1+\left|\mathrm{x}-\lambda_{\mathrm{i}}(0) \mathrm{t}\right|\right)^{1+\mu}\left|\mathrm{v}_{\mathrm{i}}(\mathrm{t}, \mathrm{x})\right| \leq C_{45}\left\{\mathrm{~V}\left(\mathrm{D}_{-}^{T}\right)+\mathrm{W}_{\infty}^{c}(\mathrm{~T})\left[\tilde{\mathrm{V}}_{1}(\mathrm{~T})+\tilde{\mathrm{U}}_{1}(\mathrm{~T}) \mathrm{V}_{\infty}(\mathrm{T})\right]\right.$

$+\mathrm{V}_{\infty}^{c}(\mathrm{~T})\left[\tilde{\mathrm{W}}_{1}(\mathrm{~T})+\mathrm{W}_{\infty}^{c}(\mathrm{~T})+\tilde{\mathrm{V}}_{1}(\mathrm{~T})+\mathrm{V}_{\infty}^{c}(\mathrm{~T})+\tilde{\mathrm{U}}_{1}(\mathrm{~T}) \mathrm{V}_{\infty}(\mathrm{T})+\mathrm{U}_{\infty}^{c}(\mathrm{~T}) \mathrm{V}_{\infty}(\mathrm{T})\right](3.144)$

$\left.+\mathrm{U}_{\infty}^{c}(\mathrm{~T}) \mathrm{V}_{\infty}(\mathrm{T})\left[\mathrm{W}_{\infty}^{c}(\mathrm{~T})+\tilde{\mathrm{W}}_{1}(\mathrm{~T})+\tilde{\mathrm{V}}_{1}(\mathrm{~T})\right]\right\}$

Then, it follows from (3.143) and (3.144) that

$V_{\infty}^{c}(\mathrm{~T}) \leq C_{46}\left\{\mathrm{~V}\left(\mathrm{D}_{-}^{T}\right)+\mathrm{W}_{\infty}^{c}(\mathrm{~T})\left[\tilde{\mathrm{V}}_{1}(\mathrm{~T})+\tilde{\mathrm{U}}_{1}(\mathrm{~T}) \mathrm{V}_{\infty}(\mathrm{T})\right]\right.$

$+\mathrm{V}_{\infty}^{c}(\mathrm{~T})\left[\tilde{\mathrm{W}}_{1}(\mathrm{~T})+\mathrm{W}_{\infty}^{c}(\mathrm{~T})+\tilde{\mathrm{V}}_{1}(\mathrm{~T})+\mathrm{V}_{\infty}^{c}(\mathrm{~T})+\tilde{\mathrm{U}}_{1}(\mathrm{~T}) \mathrm{V}_{\infty}(\mathrm{T})+\mathrm{U}_{\infty}^{c}(\mathrm{~T}) \mathrm{V}_{\infty}(\mathrm{T})\right](3.145)$

$\left.+\mathrm{U}_{\infty}^{c}(\mathrm{~T}) \mathrm{V}_{\infty}(\mathrm{T})\left[\mathrm{W}_{\infty}^{c}(\mathrm{~T})+\tilde{\mathrm{W}}_{1}(\mathrm{~T})+\tilde{\mathrm{V}}_{1}(\mathrm{~T})\right]\right\}$

We next estimate $\tilde{\mathrm{U}}_{1}$ and $U 1(T)$.

For $i=m+1 ;:: ; ; n$, for any given $\mathrm{jth}$ characteristic $\tilde{C}_{j} \operatorname{In} D_{i}^{T}(\mathrm{j} \neq \mathrm{i})$ as in the proof of (3.90), in order to estimate $\tilde{U}_{1}(T)$ it suffices to estimate

$$
\int_{0}^{y_{1}}\left|\mathrm{Z}_{\mathrm{i}}\left(\mathrm{t}, \tilde{\mathrm{x}}_{\mathrm{i}}(\mathrm{t}, \mathrm{y})\right)\right|_{t=t(\mathrm{y})} d y \text { and } \int_{0}^{y_{2}}\left|\mathrm{Z}_{\mathrm{i}}\left(\mathrm{t}, \tilde{\mathrm{x}}_{\mathrm{i}}(\mathrm{t}, \mathrm{y})\right)\right|_{t=t(\mathrm{y})} d y
$$

By integrating (2.39) along $\xi=\tilde{x}_{i}(\mathrm{~s}, \mathrm{y})$ noting (2.41), similar to (3.84), we have

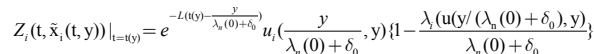

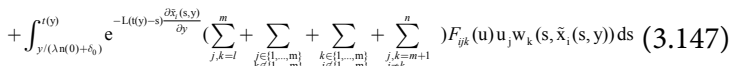

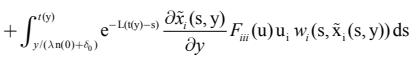

Since $\lambda i(u)$ is weakly linearly degenerate and $u=(u 1 ;:: ; ; u n) T$ are normalized coordinates, by (2.43),

we have

$$
F_{i i i}\left(\mathrm{u}_{\mathrm{i}} \mathrm{e}_{\mathrm{i}}\right) \equiv 0, \forall\left|\mathrm{u}_{\mathrm{i}}\right|
$$

Then, using Hadamard's formula, we have

$$
\begin{aligned}
F_{i i i}(\mathrm{u}) & =\mathrm{F}_{\mathrm{iii}}(\mathrm{u})-\mathrm{F}_{\mathrm{iii}}\left(\mathrm{u}_{\mathrm{i}} \mathrm{e}_{\mathrm{i}}\right) \\
& =\int_{0}^{1} \sum_{l \neq i} \frac{\partial F_{i i i}}{\partial u_{l}}\left(\mathrm{Tu}_{1}, \ldots, \mathrm{Tu}_{\mathrm{i}-1}, \mathrm{u}_{\mathrm{i}}, \mathrm{Tu}_{\mathrm{i}+1}, \ldots, \mathrm{Tu}_{\mathrm{n}}\right) \mathrm{u}_{1} \mathrm{~d}_{\mathrm{T}}
\end{aligned}
$$

Hence, noting (3.6), (3.11), (3.13) and the fact that $L>0$ and $\frac{\partial \tilde{x}_{i}(\mathrm{~s}, \mathrm{y})}{\partial y}>0$ we obtain from (3.147) and (3.149) that

$\left.z_{i}\left(\mathrm{t}, \tilde{\mathrm{x}}_{\mathrm{i}}(\mathrm{t}, \mathrm{y})\right)\right|_{\mathrm{t}=(\mathrm{y})} \leq \mathrm{u}_{\mathrm{i}}\left(\frac{y}{\lambda_{n}(0)+\delta_{0}}, \mathrm{y}\right) \mid+\mathrm{C}_{47}\left\{\left[\mathrm{~W}_{\infty}^{c}(\mathrm{~T}) \mathrm{U}_{\infty}^{c}(\mathrm{~T})+\mathrm{W}_{\infty}^{c}(\mathrm{~T}) \mathrm{U}_{\infty}^{c}(\mathrm{~T}) \mathrm{V}_{\infty}(\mathrm{T})\right]\right.$

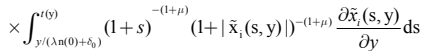

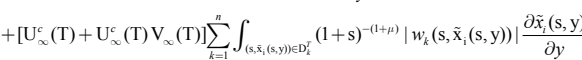

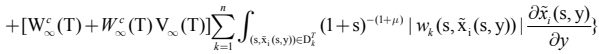

By Lemma 3.2, similar to (3.88), it follows from (3.150) that

$\int_{0}^{y 2}\left|\mathrm{Zi}\left(\mathrm{t}, \tilde{\mathrm{x}}_{\mathrm{i}}(\mathrm{t}, \mathrm{y})\right)\right|_{t=t(\mathrm{y})} \leq C_{48}\left(\theta+\mathrm{W}_{\infty}^{c}(\mathrm{~T}) \mathrm{U}_{\infty}^{c}(\mathrm{~T})+\mathrm{U}_{\infty}^{c}(\mathrm{~T}) \mathrm{W}_{1}(\mathrm{~T})+\mathrm{W}_{\infty}^{c}(\mathrm{~T}) \mathrm{U}_{1}(\mathrm{~T})(3.151)\right.$ $\left.+\left[\mathrm{W}_{\infty}^{c}(\mathrm{~T}) \mathrm{U}_{\infty}^{c}(\mathrm{~T})+\mathrm{U}_{\infty}^{c}(\mathrm{~T})+\mathrm{W}_{\infty}^{c}(\mathrm{~T}) \mathrm{U}_{1}(\mathrm{~T})\right] \mathrm{V}_{\infty}(\mathrm{T})\right\}$

Similarly, we have

$\int_{0}^{y 2}\left|\mathrm{Zi}\left(\mathrm{t}, \tilde{\mathrm{x}}_{\mathrm{i}}(\mathrm{t}, \mathrm{y})\right)\right|_{t=(y)} \leq C_{49}\left\{\mathrm{~V}_{(\mathrm{D}}\left(\mathrm{D}_{-}^{T}\right)+\mathrm{W}_{\infty}^{c}(\mathrm{~T}) \mathrm{U}_{\infty}^{c}(\mathrm{~T})+\mathrm{U}_{\infty}^{c}(\mathrm{~T}) \mathrm{W}_{1}(\mathrm{~T})+\mathrm{W}_{\infty}^{c}(\mathrm{~T}) \mathrm{U}_{1}(\mathrm{~T})\right.$ $\left.+\left[\mathrm{W}_{\infty}^{c}(\mathrm{~T}) \mathrm{U}_{\infty}^{c}(\mathrm{~T})+\mathrm{U}_{\infty}^{c}(\mathrm{~T})+\mathrm{W}_{\infty}^{c}(\mathrm{~T}) \mathrm{U}_{1}(\mathrm{~T})\right] \mathrm{V}_{\infty}(\mathrm{T})\right\}$

Thus, we obtain

$\tilde{U}_{1}(\mathrm{~T}) \leq C_{50}\left\{\theta+\mathrm{V}\left(\mathrm{D}_{-}^{T}\right)+\mathrm{W}_{\infty}^{c}(\mathrm{~T}) \mathrm{U}_{\infty}^{c}(\mathrm{~T})+\mathrm{U}_{\infty}^{c}(\mathrm{~T}) \mathrm{W}_{1}(\mathrm{~T})+\mathrm{W}_{\infty}^{c}(\mathrm{~T}) \mathrm{U}_{1}(\mathrm{~T})\right.$ $\left.+\left[\mathrm{W}_{\infty}^{c}(\mathrm{~T}) \mathrm{U}_{\infty}^{c}(\mathrm{~T})+\mathrm{U}_{\infty}^{c}(\mathrm{~T})+\mathrm{W}_{\infty}^{c}(\mathrm{~T}) \mathrm{U}_{1}(\mathrm{~T})\right] \mathrm{V}_{\infty}(\mathrm{T})\right\}$

Similarly, we have

$U_{1}(\mathrm{~T}) \leq C_{51}\left\{\theta+\mathrm{V}\left(\mathrm{D}_{-}^{T}\right)+\mathrm{W}_{\infty}^{c}(\mathrm{~T}) \mathrm{U}_{\infty}^{c}(\mathrm{~T})+\mathrm{U}_{\infty}^{c}(\mathrm{~T}) \mathrm{W}_{1}(\mathrm{~T})+\mathrm{W}_{\infty}^{c}(\mathrm{~T}) \mathrm{U}_{1}(\mathrm{~T})\right.$ $\left.+\left[\mathrm{W}_{\infty}^{c}(\mathrm{~T}) \mathrm{U}_{\infty}^{c}(\mathrm{~T})+\mathrm{U}_{\infty}^{c}(\mathrm{~T})+\mathrm{W}_{\infty}^{c}(\mathrm{~T}) \mathrm{U}_{1}(\mathrm{~T})\right] \mathrm{V}_{\infty}(\mathrm{T})\right\}$

We next estimate $U_{\infty}^{c}$

(i) For $r=1 ;:: ; ; m$, for any fixed point $(\mathrm{t}, \mathrm{x}) \in \mathrm{D}^{T}$ noting (2.19) and (2.20), similar to (3.98), we have

$$
\begin{aligned}
& u_{r}(\mathrm{t}, \mathrm{x})=\mathrm{e}^{-\mathrm{L}\left(\mathrm{t}-\mathrm{t}_{0}\right)} u_{r}\left(\mathrm{t}_{0}, \mathrm{y}\right) \\
& +\int_{t_{0}}^{t} \mathrm{e}^{-\mathrm{L}(\mathrm{t}-\mathrm{s})}\left(\sum_{i, l=l}^{m}+\sum_{\substack{i \in\{1, \ldots, \mathrm{m}\} \\
l \notin\{1, \ldots, \mathrm{m}\}}}+\sum_{\substack{l \in\left\{1, \ldots, \ldots, \mathrm{m}_{\}}\right\} \\
i \notin\{i, \ldots, \mathrm{m}\}}}+\sum_{\substack{\mathrm{l}, \mathrm{i}=m+1 \\
i \neq k}}^{n}\right) \rho_{r i l}(\mathrm{u}) \mathrm{u}_{\mathrm{j}} \mathrm{w}_{\mathrm{k}}\left(\mathrm{s}, \tilde{\mathrm{x}}_{\mathrm{i}}(\mathrm{s}, \mathrm{y})\right) \mathrm{ds}
\end{aligned}
$$

By using Lemma 3.2 and noting (3.93) and (3.96), it is easy to see that $\left|\mathrm{u}_{\mathrm{r}}\left(\mathrm{t}_{0}, \mathrm{y}\right)\right| \leq \mathrm{C}_{52} \theta(1+\mathrm{y})^{-(1+\mu)} \leq C_{53} \theta\left(1+t_{0}\right)^{-(1+\mu)} \leq C_{54} \theta(1+\mathrm{t})^{-(1+\mu)}(3.156)$ Thus, noting (3.93) and the fact that $L>0$, we obtain from (3.155) that $(1+\mathrm{t})^{1+\mu}\left|\mathrm{u}_{\mathrm{r}}(\mathrm{t}, \mathrm{x})\right| \leq \mathrm{C}_{55}\left\{\theta+\mathrm{W}_{\infty}^{c}(\mathrm{~T}) \mathrm{U}_{\infty}^{c}(\mathrm{~T})+U_{\infty}^{c}(\mathrm{~T}) \tilde{\mathrm{W}}_{1}(\mathrm{~T})=\mathrm{W}_{\infty}^{c}(\mathrm{~T}) \tilde{\mathrm{U}}_{1}(\mathrm{~T})\right\}(3.157)$

(ii) For $i=m+1 ;:: ; ; n$, for any fixed point $(\mathrm{t}, \mathrm{x}) \in \mathrm{D}^{T}$ but $(\mathrm{t}, \mathrm{x}) \notin \mathrm{D}_{i}^{T}$ similar to (3.116), we have

$\left(1+\left|\mathrm{x}-\lambda_{i}(0) \mathrm{t}\right|\right)^{1+\mu}\left|\mathrm{u}_{\mathrm{r}}(\mathrm{t}, \mathrm{x})\right| \leq \mathrm{C}_{56}\left\{\theta+\mathrm{W}_{\infty}^{c}(\mathrm{~T}) \mathrm{U}_{\infty}^{c}(\mathrm{~T})+U_{\infty}^{c}(\mathrm{~T}) \tilde{\mathrm{W}}_{1}(\mathrm{~T})=\mathrm{W}_{\infty}^{c}(\mathrm{~T}) \tilde{\mathrm{U}}_{1}(\mathrm{~T})\right\}(3.158)$

Then, it follows from (3.157) and (3.158) that

$U_{\infty}^{c} \leq \mathrm{C}_{57}\left\{\theta+\mathrm{W}_{\infty}^{c}(\mathrm{~T}) \mathrm{U}_{\infty}^{c}(\mathrm{~T})+U_{\infty}^{c}(\mathrm{~T}) \tilde{\mathrm{W}}_{1}(\mathrm{~T})=\mathrm{W}_{\infty}^{c}(\mathrm{~T}) \tilde{\mathrm{U}}_{1}(\mathrm{~T})\right\}$

We now estimate $V_{\infty}(\mathrm{T})$

For $i=m+1 ;::: ; n$, passing through any given point $(\mathrm{t}, \mathrm{x}) \in \mathrm{D}^{T}$ we draw the ith characteristic $\xi=\xi_{i}(\mathrm{~s} ; \mathrm{t}, \mathrm{x})$ which intersects one of the boundaries of $D T$ at one point. For fixing the idea, suppose that this characteristic intersects $x=\left(\lambda_{\mathrm{n}}(0)+\delta_{0}\right)$ t at a point $\left(\mathrm{y} /\left(\lambda_{\mathrm{n}}(0)+\delta_{0}\right), \mathrm{y}\right)$ 
By integrating (2.13) along this characteristic and noting (2.16)-(2.18), we have

$$
\begin{aligned}
& V r(\mathrm{t}, \mathrm{x})=\mathrm{e}^{-\mathrm{L}\left(\mathrm{t}-\mathrm{t}_{0}\right)} V r\left(\mathrm{t}_{0}, \mathrm{y}\right)
\end{aligned}
$$

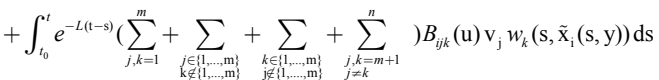

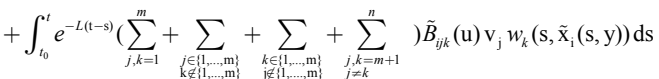

$$
\begin{aligned}
& +\int_{t_{0}}^{t} e^{-L(t-s)}\left[\sum_{l=m+1}^{n}\left(\tilde{\beta}_{\mathrm{rll}}(\mathrm{u})-\tilde{\beta}_{\mathrm{rll}}\left(\mathrm{u}_{1} \mathrm{e}_{1}\right)\right) \mathrm{v}_{l}^{2}\right](\mathrm{s}, \xi \mathrm{r}(\mathrm{s} ; \mathrm{t}, \mathrm{x})) \mathrm{ds} \\
& +\int_{t_{0}}^{t} e^{-L(t-s)}\left[\sum_{l=m+1}^{n}\left(\tilde{\beta}_{\mathrm{rll}}(\mathrm{u})-\tilde{\beta}_{\mathrm{rll}}\left(\mathrm{u}_{1} \mathrm{e}_{1}\right)\right) \mathrm{v}_{l}^{2}\right](\mathrm{s}, \xi \mathrm{r}(\mathrm{s} ; \mathrm{t}, \mathrm{x})) \mathrm{ds}
\end{aligned}
$$

Noting Lemma 3.1 and Lemma 3.2, and using Hadamard's formula, it follows from (3.160) that

$$
\begin{aligned}
& \left|V_{i}(\mathrm{t}, \mathrm{x})\right| \leq C_{58}\left\{\theta+\mathrm{V}\left(\mathrm{D}_{-}^{T}\right)+\mathrm{W}_{\infty}^{c}(\mathrm{~T}) \mathrm{U}_{\infty}^{c}(\mathrm{~T})+\mathrm{U}_{\infty}^{c}(\mathrm{~T}) \mathrm{W}_{1}(\mathrm{~T})+\mathrm{W}_{\infty}^{c}(\mathrm{~T}) \mathrm{U}_{1}(\mathrm{~T})\right. \\
& \left.+\left[\mathrm{W}_{\infty}^{c}(\mathrm{~T}) \mathrm{U}_{\infty}^{c}(\mathrm{~T})+\mathrm{U}_{\infty}^{c}(\mathrm{~T})+\mathrm{W}_{\infty}^{c}(\mathrm{~T}) \mathrm{U}_{1}(\mathrm{~T})\right] \mathrm{V}_{\infty}(\mathrm{T})\right\}
\end{aligned}
$$

On the other hand, for $i=m+1 ;::: ; n$, for any fixed point $(\mathrm{t}, \mathrm{x}) \notin \mathrm{D}(\mathrm{T})=\{(\mathrm{t}, \mathrm{x}) \mid 0 \leq \mathrm{t} \leq \mathrm{T}, \mathrm{x} \geq 0\} \quad$ but $\quad(\mathrm{t}, \mathrm{x}) \notin \mathrm{D}_{i}^{T},\left|\mathrm{v}_{\mathrm{i}}(\mathrm{t}, \mathrm{x})\right|$ can be controlled by $V_{\infty}^{c}(\mathrm{~T})$ or $\mathrm{V}\left(\mathrm{D}_{ \pm}^{T}\right)$ Moreover, for $i=1 ;:: ; m$, for any fixed point $(\mathrm{t}, \mathrm{x}) \notin \mathrm{D}(\mathrm{T})=\{(\mathrm{t}, \mathrm{x}) \mid 0<\mathrm{t}<\mathrm{T}, \mathrm{x} \geq 0\}\left|\mathrm{v}_{\mathrm{i}}(\mathrm{t}, \mathrm{x})\right|$ can be controlled by $V_{\infty}^{c}(\mathrm{~T})$ or $\mathrm{V}\left(\mathrm{D}_{ \pm}^{T}\right)$ as well. Thus, by using Lemma 3.2 again, we have

$$
\begin{aligned}
& V_{\infty} \leq C_{58}\left\{\theta+\mathrm{V}\left(\mathrm{D}_{-}^{T}\right)+\mathrm{W}_{\infty}^{c}(\mathrm{~T}) \mathrm{U}_{\infty}^{c}(\mathrm{~T})+\mathrm{U}_{\infty}^{c}(\mathrm{~T}) \mathrm{W}_{1}(\mathrm{~T})+\mathrm{W}_{\infty}^{c}(\mathrm{~T}) \mathrm{U}_{1}(\mathrm{~T})\right. \\
& \left.+\left[\mathrm{W}_{\infty}^{c}(\mathrm{~T}) \mathrm{U}_{\infty}^{c}(\mathrm{~T})+\mathrm{U}_{\infty}^{c}(\mathrm{~T})+\mathrm{W}_{\infty}^{c}(\mathrm{~T}) \mathrm{U}_{1}(\mathrm{~T})\right] \mathrm{V}_{\infty}(\mathrm{T})\right\}
\end{aligned}
$$

We finally estimate $W_{\infty}(\mathrm{T})$

For $i=m+1 ;::: ; n$, passing through any given point $(\mathrm{t}, \mathrm{x}) \in \mathrm{D}^{T}$ similar to (3.160), noting (2.9), (2.11)-(2.12) and the fact that $\lambda_{i}(\mathrm{u})$ is weakly linearly degenerate, we have

$$
\begin{aligned}
& \left.w_{i}(\mathrm{t}, \mathrm{x})=e^{-L\left(t(\mathrm{y})-\frac{y}{\lambda_{n}(0)+\delta_{0}}\right.}\right) w_{i}\left(\frac{y}{\lambda_{n}(0)+\delta_{0}}, \mathrm{y}\right)
\end{aligned}
$$

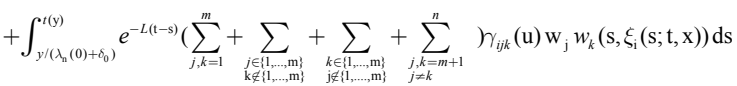

$$
\begin{aligned}
& \left.+\int_{y /\left(\lambda_{i}(0)+\delta_{0}\right)}^{t} e^{-L(t(y)-s)}\left[\gamma_{i i i}(\mathrm{u})-\gamma_{i i i}\left(\mathrm{u}_{\mathrm{i}} \mathrm{e}_{\mathrm{i}}\right)\right) \mathrm{w}_{i}^{2}\right]\left(\mathrm{s}, \xi_{\mathrm{i}}(\mathrm{s} ; \mathrm{t}, \mathrm{x})\right) \mathrm{ds}
\end{aligned}
$$

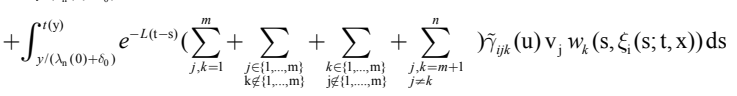

$$
\begin{aligned}
& \left.+\int_{y /\left(\lambda_{\mathrm{x}}(0)+\delta_{0}\right)}^{t(\mathrm{y})} e^{-L(\mathrm{t}-\mathrm{s})}\left[\sum_{j=m+1}^{n} \tilde{\gamma}_{i j}(\mathrm{u})-\tilde{\gamma}_{i i i}\left(\mathrm{u}_{\mathrm{i}} \mathrm{e}_{\mathrm{i}}\right)\right) \mathrm{w}_{i}^{2}\right]\left(\mathrm{s}, \xi_{\mathrm{i}}(\mathrm{s} ; \mathrm{t}, \mathrm{x})\right) \mathrm{ds}
\end{aligned}
$$

Noting Lemma 3.1 and Lemma 3.2, and using Hadamard's formula, it follows from (3.163) that

$$
\begin{aligned}
& \left|\mathrm{w}_{\mathrm{i}}(\mathrm{t}, \mathrm{x})\right| \leq C_{60}\left\{\theta+\mathrm{W}_{\infty}^{c}(\mathrm{~T}) \mathrm{U}_{\infty}^{c}(\mathrm{~T})+\mathrm{U}_{\infty}^{c}(\mathrm{~T}) \mathrm{W}_{1}(\mathrm{~T})+\mathrm{W}_{\infty}^{c}(\mathrm{~T}) \mathrm{U}_{1}(\mathrm{~T})\right. \\
& \left.+\left[\mathrm{W}_{\infty}^{c}(\mathrm{~T}) \mathrm{U}_{\infty}^{c}(\mathrm{~T})+\mathrm{U}_{\infty}^{c}(\mathrm{~T})+\mathrm{W}_{\infty}^{c}(\mathrm{~T}) \mathrm{U}_{1}(\mathrm{~T})\right] \mathrm{V}_{\infty}(\mathrm{T})\right\}
\end{aligned}
$$

Thus, by using the definitions of $W_{\infty}^{c}(\mathrm{~T}), \mathrm{W}\left(\mathrm{D}_{+}^{T}\right)$ and $\mathrm{W}\left(\mathrm{D}_{-}^{T}\right)$ and using Lemma 3.2, we have

$W_{\infty}(\mathrm{T}) \leq C_{61}\left\{\theta+W\left(\mathrm{D}_{-}^{T}\right)+\mathrm{W}_{\infty}^{c}(\mathrm{~T}) \mathrm{U}_{\infty}^{c}(\mathrm{~T})+\mathrm{U}_{\infty}^{c}(\mathrm{~T}) \mathrm{W}_{1}(\mathrm{~T})+\mathrm{W}_{\infty}^{c}(\mathrm{~T}) \mathrm{U}_{1}(\mathrm{~T})\right.$

$\left.+\left[\mathrm{W}_{\infty}^{c}(\mathrm{~T}) \mathrm{U}_{\infty}^{c}(\mathrm{~T})+\mathrm{U}_{\infty}^{c}(\mathrm{~T})+\mathrm{W}_{\infty}^{c}(\mathrm{~T}) \mathrm{U}_{1}(\mathrm{~T})\right] \mathrm{V}_{\infty}(\mathrm{T})\right\}$

We now prove (3.47)-(3.53).

Noting (1.15), evidently we have

$$
\begin{aligned}
& W_{\infty}^{c}(0), \mathrm{V}_{\infty}^{c}(0), \mathrm{U}_{\infty}^{c}(0) \leq \mathrm{C}_{62} \theta \\
& W_{1}(0)=\mathrm{V}_{1}(0)=\mathrm{U}_{1}(0)=\tilde{\mathrm{W}}_{1}(0)=\tilde{\mathrm{V}}_{1}(0)=\tilde{\mathrm{U}}_{1}(0)=0
\end{aligned}
$$

$$
W_{\infty}(0), \mathrm{V}_{\infty}(0) \leq \mathrm{C}_{63} \theta
$$

And

$$
T=0: W\left(\mathrm{D}_{-}^{T}\right), \mathrm{V}\left(\mathrm{D}_{-}^{T}\right) \leq \mathrm{C}_{64} \theta
$$

Thus, by continuity there exist positive constants $k 2$; $k 3 ; k 4 ; k 5 ; k 6$; $k 7$ and $k 8$ independent of $\mu$, such that (3.47)-(3.53) hold at least for $0 \leq T \leq T 0$ whereT0 is a small positive number. Hence, in order to prove (3.47)-(3.53) it su \pm ces to show that we can choose $k 2 ; k 3 ; k 4 ; k 5$; $k 6 ; k 7$ and $k 8$ in such a way that for any fixed $T_{0}\left(0<T_{0} \leq \mathrm{T}\right)$.

$$
\begin{aligned}
& W\left(\mathrm{D}_{-}^{T_{0}}\right) \leq 2 \mathrm{k}_{2} \theta \\
& \mathrm{V}\left(\mathrm{D}_{-}^{T_{0}}\right) \leq 2 \mathrm{k}_{3} \theta \\
& \mathrm{U}_{\infty}^{c}\left(\mathrm{~T}_{0}\right) \leq 2 \mathrm{k}_{4} \theta \\
& W_{\infty}^{c}\left(\mathrm{~T}_{0}\right), \mathrm{V}_{\infty}^{c}\left(\mathrm{~T}_{0}\right) \leq 2 \mathrm{k}_{5} \theta \\
& \tilde{W}_{1}\left(\mathrm{~T}_{0}\right), \mathrm{W}_{1}\left(\mathrm{~T}_{0}\right), \tilde{\mathrm{V}}_{1}\left(\mathrm{~T}_{0}\right), \mathrm{V}_{1}\left(\mathrm{~T}_{0}\right), \tilde{\mathrm{U}}_{1}\left(\mathrm{~T}_{0}\right), U_{1}\left(\mathrm{~T}_{0}\right) \leq 2 \mathrm{k}_{6} \theta \\
& \mathrm{V}_{\infty}\left(\mathrm{T}_{0}\right), \mathrm{U}_{\infty}\left(\mathrm{T}_{0}\right) \leq 2 \mathrm{k}_{7} \theta \\
& \mathrm{W}_{\infty}\left(\mathrm{T}_{0}\right) \leq 2 \mathrm{k}_{8} \theta \\
& \text { we have } \\
& W\left(\mathrm{D}_{-}^{T_{0}}\right) \leq \mathrm{k}_{2} \theta \\
& W\left(\mathrm{D}_{-}^{T_{0}}\right) \leq \mathrm{k}_{3} \theta \\
& W\left(\mathrm{D}_{-}^{T_{0}}\right) \leq \mathrm{k}_{4} \theta \\
& W_{\infty}^{c}\left(\mathrm{~T}_{0}\right), \mathrm{V}_{\infty}^{c}\left(\mathrm{~T}_{0}\right) \leq \mathrm{k}_{5} \theta \\
& \tilde{W}_{1}\left(\mathrm{~T}_{0}\right), \mathrm{W}_{1}\left(\mathrm{~T}_{0}\right), \tilde{\mathrm{V}}_{1}\left(\mathrm{~T}_{0}\right), \mathrm{V}_{1}\left(\mathrm{~T}_{0}\right), \tilde{\mathrm{U}}_{1}\left(\mathrm{~T}_{0}\right), U_{1}\left(\mathrm{~T}_{0}\right) \leq \mathrm{k}_{6} \theta \\
& V\left(\mathrm{~T}_{0}\right), \mathrm{U}_{\infty}\left(\mathrm{T}_{0}\right) \leq \mathrm{k}_{7} \theta \\
& W\left(\mathrm{~T}_{0}\right) \leq \mathrm{k}_{8} \theta
\end{aligned}
$$

To this end, substituting (3.170)-(3.176) into the right-hand sides of (3.79), (3.90), (3.92), (3.117), (3.129), (3.137)-(3.138), (3.145), (3.153)-(3.154), (3.159), (3.162) and (3.165)(in which we take $T=T 0)$, it is easy to see that, when $\theta_{0}>0$ is suitably small, we have

$$
\begin{aligned}
& W\left(\mathrm{D}_{-}^{T_{0}}\right) \leq 2 \mathrm{C}_{13}\left(1+\mathrm{k}_{3}\right) \theta \\
& \tilde{W}_{1}\left(\mathrm{D}_{-}^{T_{0}}\right) \leq 2 \mathrm{C}_{13}\left(1+\mathrm{k}_{2}\right) \theta \\
& W_{1}\left(\mathrm{~T}_{0}\right) \leq 2 \mathrm{C}_{19}\left(1+\mathrm{k}_{2}\right) \theta \\
& W_{\infty}^{c}\left(\mathrm{~T}_{0}\right) \leq 2 \mathrm{C}_{29}\left(1+\mathrm{k}_{2}\right) \theta \\
& V\left(\mathrm{D}_{-}^{T_{0}}\right) \leq 2 \mathrm{C}_{37} \theta \\
& \tilde{\mathrm{V}}_{1}\left(\mathrm{~T}_{0}\right) \leq 2 \mathrm{C}_{40}\left(1+\mathrm{k}_{3}\right) \theta \\
& \mathrm{V}_{1}\left(\mathrm{~T}_{0}\right) \leq 2 \mathrm{C}_{41}\left(1+\mathrm{k}_{3}\right) \theta \\
& \mathrm{V}_{\infty}^{c}\left(\mathrm{~T}_{0}\right) \leq 2 \mathrm{C}_{46}\left(1+\mathrm{k}_{3}\right) \theta \\
& \tilde{\mathrm{U}}_{1}\left(\mathrm{~T}_{0}\right) \leq 2 \mathrm{C}_{50}\left(1+\mathrm{k}_{3}\right) \theta \\
& \mathrm{U}_{1}\left(\mathrm{~T}_{0}\right) \leq 2 \mathrm{C}_{51}\left(1+\mathrm{k}_{3}\right) \theta \\
& \mathrm{U}_{\infty}^{c}\left(\mathrm{~T}_{0}\right) \leq 2 \mathrm{C}_{57}\left(1+\mathrm{k}_{3}\right) \theta
\end{aligned}
$$


Citation: Shao ZQ (2014) Global Classical Solutions to the Mixed Initial-boundary Value Problem for a Class of Quasilinear Hyperbolic Systems of Balance Laws. J Appl Computat Math 4: 197. doi:10.4172/2168-9679.1000197

Page 13 of 14

$$
\begin{aligned}
& \mathrm{V}_{\infty}\left(\mathrm{T}_{0}\right) \leq 2 \mathrm{C}_{59}\left(1+\mathrm{k}_{3}+\mathrm{k}_{5}\right) \theta \\
& W_{\infty}\left(\mathrm{T}_{0}\right) \leq 2 \mathrm{C}_{61}\left(1+\mathrm{k}_{2}+\mathrm{k}_{5}\right) \theta
\end{aligned}
$$

Hence, if $k 3 \geq 2 C 37 ; \quad k 2 \geq 2 C 13(1+k 3) ; \quad k 4 \geq 2 C 57(1+k 3) ; \quad k 5 \geq 2$ $\max f C 29(1+k 2) ; C 46(1+k 3) g, \quad k 6 \geq 2 \max f C 18(1+k 2) ; C 19(1+k 2) ; \quad C 40$ $(1+k 3) ; C 41(1+k 3) ; C 50(1+k 3) ; C 51(1+k 3) g, \quad k 7 \geq 2 C 59(1+k 3+k 5) \quad$ and $k 8 \geq 2 C 61(1+k 2+k 5)$, then we get (3.177)-(3.183). This proves (3.47)(3.53). have

Finally, we observe that when $\mu 0>0$ is suitably small, by (3.52) we

$$
U_{\infty}(\mathrm{T}) \leq \mathrm{k}_{7} \theta \leq \mathrm{k}_{7} \theta \leq \frac{\delta}{2}
$$

This implies the validity of hypothesis (3.6). The proof of Lemma 3.3 is finished.

Proof of Theorem 1.1. It su \pm ces to prove Theorem 1.1 in the normalized coordinates. Under the assumptions of Theorem 1.1, by (3.52) and (3.53), we know that there is a su \pm ciently small $\theta_{0}>0$ such that for any fixed $\theta \in\left(0, \theta_{0}\right]$ on any given domain of existence $D(\mathrm{~T})=\{(\mathrm{t}, \mathrm{x}) \mid 0 \leq \mathrm{t} \leq \mathrm{T}, \mathrm{x} \geq 0\}$ of the $C 1$ solution $u=u(t ; x)$ to the mixed initial-boundary value problem (1.1) and (1.12)-(1.13), we have the following uniform a priori estimate for the $C 1$ norm of the solution:

$$
\|u(\mathrm{t}, .)\| \mathrm{c}^{1} \triangleq\|u(\mathrm{t}, .)\| \mathrm{c}^{0}+\left\|\mathrm{u}_{\mathrm{x}}(\mathrm{t}, .)\right\| \mathrm{c}^{0} \leq k_{9} \theta
$$

Thus we immediately get the conclusion of Theorem 1.1 . The proof of Theorem 1.1 is finished.

\section{Application}

Consider the following mixed initial-boundary value problem for the system of the flow equations of a model class of ${ }^{\circ}$ uids with viscosity induced by fading memory (cf. [7]):

$$
\left\{\begin{array}{c}
w t-v x+w=0 \\
v t-(\sigma(\mathrm{w})) \mathrm{x}+\mathrm{v}=0
\end{array}\right.
$$

with the initial condition

$$
t=0: w=w_{0}(\mathrm{x}), \mathrm{v}=\tilde{\mathrm{v}}_{0}+v_{0}(\mathrm{x})(\mathrm{x} \geq 0)
$$

and the boundary condition

$$
x=0: v=h(\mathrm{t})(\mathrm{t} \geq 0)
$$

Here, $w$ is the displacement gradient and $v$ the velocity of a model class of fluids, the stress-strain function $\sigma(w)$ is a suitably smooth function of $w$ such that

$$
\sigma^{\prime}(0)>0
$$

$\tilde{V}_{0}$ is a constant, $\left(\mathrm{W}_{0}(\mathrm{x}), \mathrm{V}_{\mathrm{o}}(\mathrm{x})\right) \in \mathrm{C}^{1}$ and we assume that there exists a constant $\mu>0$ such that

$$
\left.\operatorname{Sup}_{c \geq 0}\left\{(1+\mathrm{x})^{1+\mu}\left(\left|\mathrm{w}_{0}(\mathrm{x})\right|\right)+\left|\mathrm{v}_{0}(\mathrm{x})\right|+\left|\mathrm{v}_{0}(\mathrm{x})\right|+\left|\mathrm{w}_{0}^{\prime}(\mathrm{x})\right|+\left|\mathrm{v}_{0}^{\prime}(\mathrm{x})\right|\right)\right\}<+\infty
$$

In addition, we assume that $h(\mathrm{t}) \in \mathrm{C}^{1}$

$$
\operatorname{Sup}_{c \geq 0}\left\{(1+t)^{1+\mu}\left(|\mathrm{h}(\mathrm{t})|+\left|\mathrm{h}^{\prime}(\mathrm{t})\right|\right)\right\}<+\infty
$$

Moreover, the conditions of $C 1$ compatibility are supposed to be satis ${ }^{-}$ed at the point $(0 ; 0)$.

Let

$$
u=\left(\begin{array}{l}
w \\
v
\end{array}\right)
$$

By (4.4), it is easy to see that in a neighborhood of $u_{0}=\left(\begin{array}{c}0 \\ \tilde{v}_{0}\end{array}\right)$ system (4.1) is strictly hyperbolic and has the following two distinct real eigenvalues:

$$
\lambda_{1}(\mathrm{u})=-\sqrt{\sigma^{\prime}(\mathrm{w})}<0<\lambda_{2}(\mathrm{u})=\sqrt{\sigma^{\prime}(\mathrm{w})}
$$

The corresponding right eigenvectors are

$$
\left.r_{1}(\mathrm{u}) / /\left(\sqrt{\sigma^{\prime}(\mathrm{w})}\right)^{\mathrm{T}}, r_{2}(\mathrm{u}) / /\left(1-\sqrt{\sigma^{\prime}(\mathrm{w})}\right)\right)^{\mathrm{T}}
$$

It is easy to see that in a neighborhood of $u_{0}=\left(\begin{array}{c}0 \\ \tilde{v}_{0}\end{array}\right)$ all characteristics are linearly degenerate,

then weakly linearly degenerate, provided that

$$
\sigma^{\prime \prime}(\mathrm{w}) \equiv 0, \forall|\mathrm{w}| \quad \text { small }
$$

The corresponding left eigenvectors can be taken as

$$
l_{1}(\mathrm{u})=\left(\sqrt{\sigma^{\prime}(\mathrm{w})}, 1\right), 1_{2}(\mathrm{u})=\left(-\sqrt{\sigma^{\prime}(\mathrm{w})}, 1\right)
$$

Let

$v i=l i(u) u(i=1 ; 2)$ :

Then, the boundary condition (4.3) can be rewritten as

$x=0: \mathrm{v}_{1}+v_{2}=2 h(\mathrm{t}) \triangleq \mathrm{H}(\mathrm{t})$

By Theorem 1.1 we get

Theorem 5.1. Suppose that (4.4) and (4.10) hold. Suppose furthermore that $w 0(x) ; v 0(x)$ are all $C 1$

functions with respect to their arguments, for which there is a constant $\mu>0$ such that

$$
\begin{aligned}
& \theta \triangleq \max \left\{\operatorname { s u p } _ { x \geq 0 } \left\{(1+\mathrm{x})^{1+\mu}\left(\left|\mathrm{w}_{0}(\mathrm{x})\right|+\left|\mathrm{v}_{0}(\mathrm{x})\right|+\left|\mathrm{w}_{0}^{\prime}(\mathrm{x})\right|+\left|\mathrm{v}_{0}^{\prime}(\mathrm{x})\right|\right),\right.\right. \\
& \left.\sup _{t \geq 0}(1+\mathrm{t})^{1+\mu}\left(|\mathrm{H}(\mathrm{t})|+\left|\mathrm{H}^{\prime}(\mathrm{t})\right|\right)\right\}<+\infty
\end{aligned}
$$

Suppose finally that $h(\mathrm{t}) \in \mathrm{C}^{1}$ satisfies (4.14) and that the conditions of $C 1$ compatibility are satisfied at the point $(0 ; 0)$. Then there is a sufficiently small $\theta_{0}>0$ such that for any given $\theta \in\left[0, \theta_{0}\right]$ the mixed initial-boundary value problem (4.1)-(4.3) admits a unique global $C 1$ solution $u=u(t ; x)$ in the half space $\{(\mathrm{t}, \mathrm{x}) \mid \mathrm{t} \geq 0, x \geq 0\}$.

\section{Acknowledgement}

The author would like to thank Prof. Ta-tsien Li very much for his valuable lectures and guidance in Fuzhou in December, 2003. The author also want to thank Prof. Jiaxing Hong very much for his guidance and constant encouragement.

\section{References}

1. Bressan A (1988) Contractive metrics for nonlinear hyperbolic systems, Indiana Univ Math J: 37 409-421.

2. Bressan A (2000) Hyperbolic Systems of Conservation Laws: The Onedimensional Cauchy Problem, Oxford University Press, Oxford.

3. Chen GQ, Levermore CD, Liu TP (1994) Hyperbolic conservation laws with sti® relaxation terms and entropy, Comm. Pure Appl Math 47: 787-830.

4. Chen GQ, Wagner DH (2003) Global entropy solutions to exothermically reacting compressible Euler equations, J Di®erential Equations 191: 277-322.

5. Dafermos CM (2000) Hyperbolic Conservation Laws in Continuum Physics, Springer, Berlin. 
Citation: Shao ZQ (2014) Global Classical Solutions to the Mixed Initial-boundary Value Problem for a Class of Quasilinear Hyperbolic Systems of Balance Laws. J Appl Computat Math 4: 197. doi:10.4172/2168-9679.1000197

Page 14 of 14

6. Dafermos CM (2006) Hyperbolic systems of balance laws with weak dissipation, J Hyperbolic Di®er-ential Equations 3: 505-527.

7. Dafermos CM, Hsiao L (1982) Hyperbolic systems of balance laws with inhomogeneity and dissipation, Indiana Univ Math J 31: 471-491.

8. Hörmander L (1987) The Lifespan of Classical Solutions of Nonlinear Hyperbolic Equations, Lecture Notes in Mathematics, Springer, Berlin 1256.

9. Hsiao L, Marcati P (1988) Nonhomogeneous quasilinear hyperbolic system arising in chemical engineer-ing, Ann. Scuola Norm Sup Pisa Cl Sci 15: 65-97.

10. Hsiao L, Pan RH (1999) Initial boundary value problem for the system of compressible adiabatic ${ }^{\circ}$ ow through porous media, J Dißerential Equations $159: 280-305$.

11. Hsiao L, Pan RH (2000) The damped p-system with boundary e®ects, Contemporary Mathematics 255: 109-123.

12. Hsiao L, Tang SQ (1995) Construction and qualitative behavior of the solution of the perturbated Riemann problem for the system of one-dimensional isentropic ${ }^{\circ} \mathrm{OW}$ with damping, J. Dißerential Equations 123: 480-503.

13. John $F(1974)$ Formation of singularities in one-dimensional nonlinear wave propagation, Comm. Pure Appl Math 27: 377-405.

14. Kong DX (2000) Cauchy Problem for Quasilinear Hyperbolic Systems, MS Memoirs, Vol. 6, The Mathematical society of Japan, Tokyo.

15. Kong DX (2000) Life-span of classical solutions to quasilinear hyperbolic systems with slow decay initial data, Chinese Ann. Math. Ser. B 21: 413-440.

16. Kong DX (2002) Formation and propagation of singularities for $2 £ 2$ quasilinear hyperbolic systems, Trans Amer Math Soc 354: 3155-3179.

17. Li TT (1994) Global Classical Solutions for Quasilinear Hyperbolic Systems Research in Applied Mathematics, Wiley/Masson, New York 32.

18. Li TT, Jin Y (2001) Semi-global $C 1$ solution to the mixed initial-boundary value problem for quasi-linear hyperbolic systems, Chinese Ann Math Ser B 22 325-336.

19. Li TT, Kong DX, Zhou Y (1996) Global classical solutions for general quasilinear non-strictly hy-perbolic systems with decay initial data, Nonlinear Studies 3 : 203-229.

20. Li TT, Peng YJ (2003) The mixed initial-boundary value problem for reducible quasilinear hyperbolic systems with linearly degenerate characteristics, Nonlinear Analysis 52: 573-583.

21. Li TT, Wang LB (2005) Global classical solutions to a kind of mixed initialboundary value problem for quasilinear hyperbolic systems, Discrete and Continuous Dynamical Systems 12 : 59-78.

22. Li TT, Yu WC (1985) Boundary Value Problems for Quasilinear Hyperbolic Systems, Duke University Mathematics Series V, Duke University, Durham.

23. Li TT, Zhou Y, Kong DX (1994) Weak linear degeneracy and global classical solutions for general quasilinear hyperbolic systems, Comm Partial Dißerential Equations 19: 1263-1317.

24. Li TT, Zhou Y, Kong DX (1997) Global classical solutions for general quasilinear hyperbolic systems with decay initial data, Nonlinear Analysis TMA 28 1299-1332.

25. Liu TP (1979) Development of singularties in the nonlinear waves for quasilinea hyperbolic partial dißerential equations, J Dißerential Equations 33: 92-111.

26. Liu TP (1979) Quasilinear hyperbolic systems, Comm Math Phys 68: 141-172.

27. Luskin M (1981) On the existence of global smooth solutions for a mode equation for ${ }^{\circ}$ uid ${ }^{\circ}$ ow in a pipe, J Math Anal Appl 84: 614-630.

28. Luskin M, Temple JB, The existence of a global weak solution to the nonlinear waterhammer problem, Comm Pure Appl Math 35: 697-735.

29. Shao ZQ (2008) Global classical solutions for a class of quasilinear hyperbolic systems of balance laws, in: E. T. Ho®mann(Ed.), Progress in Nonlinear Analysis Research, Nova Science Publishers, Hauppauge, NY 285-308.

30. Shao ZQ (2008) Global solutions with shock waves to the generalized Riemann problem for a class of quasilinear hyperbolic systems of balance laws II, Math Nachr 281: 879-902.

31. Yang T, Zhu CJ, Zhao HJ (1997) Global smooth solutions for a class of quasilinear hyperbolic systems with dissipative terms, Proc Roy Soc Edinburgh Ser A 127: 1311-1324.

32. Ying LA, Wang CH (1980) Global solutions of the Cauchy problem for a nonhomogeneous quasilinear hyperbolic system, Comm. Pure Appl Math 33 579-597.

33. Ying LA, Wang $\mathrm{CH}$ (1980) Solution in the large for nonhomogeneous quasilinear hyperbolic systems of equations, J Math Anal Appl 78: 440-454. 